\title{
Environmental Evolution of Long-Lived Supercell Thunderstorms in the Great Plains
}

\author{
CASEY E. DAVENPORT ${ }^{\mathrm{a}}$ \\ ${ }^{a}$ University of North Carolina at Charlotte, Charlotte, North Carolina
}

(Manuscript received 17 March 2021, in final form 13 October 2021)

\begin{abstract}
Long-lived supercells (containing mesocyclones persisting for at least $4 \mathrm{~h}$ ) are relatively rare, but present significant risk for society as a result of their intensity and associated hazards over an extended time period. The persistence of a rotating updraft is tied to near-storm environmental characteristics; however, given the established prevalence of mesoscale environmental heterogeneity near severe convection, it is unknown to what extent those near-storm characteristics vary over the lifetime of a supercell, nor how quickly the storm responds to such changes. This study examines 147 long-lived, isolated supercells, focusing on the evolution of their near-storm environments using model analysis soundings generated each hour throughout the storm's lifetime. Environmental variability is quantified via a series of common forecasting parameters, with impacts of measured changes related to production of severe weather and overall storm longevity. The diurnal and maturity-relative distributions of forecasting parameters are examined, along with comparisons among subsets of marginally versus very long-lived supercells, as well as dissipation before versus after sunset. The diurnal cycle is a dominant trend over the lifetime of all supercells, with attendant impacts to relevant thermodynamic and kinematic parameters, timing of storm initiation and dissipation, as well as severe weather production. Notably, changes in the near-storm environment are connected to supercell longevity and generation of severe weather reports. The long-term goal of the above analyses is to enhance short-term forecasts of supercells by better anticipating storm evolution as a result of environmental variations.
\end{abstract}

SIGNIFICANCE STATEMENT: The environments in which severe thunderstorms occur often vary substantially over time and space. To understand how these changes influence the evolution and severe weather production of supercell thunderstorms, the near-storm environments of 147 long-lived supercells were examined using hourly weather model data. Daily patterns of daytime heating and cooling, along with associated changes to the low-level wind profile, dominated the near-storm environmental trends. However, environmental changes appear to be important in distinguishing very long-lived supercells from marginally long-lived supercells, and are also relevant to the production of severe weather. These findings have implications for being able to forecast supercell maintenance and development of tornadoes, large hail, or severe wind gusts.

KEYWORDS: Severe storms; Supercells; Mesoscale forecasting

\section{Introduction}

Long-lived supercell thunderstorms, defined by Bunkers et al. (2006a) as those persisting in a quasi-steady manner for $\geq 4 \mathrm{~h}$, present significant hazards to the general public over an extended period of time. For example, when compared to short-lived supercells (lifetimes $<2 \mathrm{~h}$ ), long-lived supercells are much more likely to produce strong or violent tornadoes (F/EF2 and above), as well as significant hail or wind reports (Bunkers et al. 2006a); at times, these reports can extend over substantial distances (e.g., Thompson and Edwards 2000; Glass and Britt 2002; Changnon and Burroughs 2003; Bosart et al. 2006).

Environments supportive of long-lived supercells tend to be characterized by significant deep-layer bulk wind shear ( $>26 \mathrm{~m} \mathrm{~s}^{-1}$ in the $0-8$-km layer), strong deep-layer stormrelative winds $\left(>17 \mathrm{~m} \mathrm{~s}^{-1}\right.$ in the $0-8$-km layer), and low-level characteristics consistent with production of significant

Corresponding author: Casey Davenport, casey.davenport@ uncc.edu tornadoes, including lower mixed-layer lifting condensation levels $(<2000 \mathrm{~m})$ and stronger (on average, $75 \mathrm{~m}^{2} \mathrm{~s}^{-2}$ higher) 0-1-km storm-relative helicity (Bunkers et al. 2006b). Furthermore, long-lived supercells tend to be much more isolated and discrete than their shorter-lived counterparts, favoring motion within a warm sector or along a boundary or moisture/buoyancy axis (Bunkers et al. 2006a,b). This observation is consistent with other research demonstrating that significant tornadoes tend to form near surface boundaries (e.g., Maddox et al. 1980; Markowski et al. 1998a; Rasmussen et al. 2000; Gagne et al. 2012; Boustead et al. 2013; Magee and Davenport 2020). The diurnal cycle and heating are influencing factors for instability and are associated with supercell formation and maintenance; long-lived supercells most frequently occur around 0000-0100 UTC (in the late afternoon and early evening, 1-2 $\mathrm{h}$ prior to sunset), with an average initiation time near 2200 UTC (i.e., midafternoon; Bunkers et al. 2006a).

Much of the above information regarding long-lived supercell environments was established by Bunkers et al. (2006a,b), largely using observed soundings within $185 \mathrm{~km}$ and $3 \mathrm{~h}$ of storm initiation (one per event, which could encompass 
multiple supercells). However, numerous studies have documented the spatial and temporal variability present within supercell environments, often occurring at comparatively smaller distances and shorter time scales (e.g., Brooks et al. 1996; Markowski et al. 1998b; Markowski and Richardson 2007; Parker 2014; Davenport and Parker 2015b; Klees et al. 2016; Gropp and Davenport 2018). Notably, as a result of such thermodynamic and kinematic variability, there have been demonstrated impacts to convective organization, storm longevity, and production of severe weather (e.g., Markowski et al. 1998a; Richardson et al. 2007; Ziegler et al. 2010; Davenport and Parker 2015a; Klees et al. 2016; Koch et al. 2016; Gropp and Davenport 2018; Hartigan et al. 2021), in line with established connections between convective parameters and forecasting severe hazards (e.g., Rasmussen and Blanchard 1998; Rasmussen 2003). Indeed, accounting for environmental variability in models leads to more realistic depiction of convection and its evolution (e.g., Ziegler et al. 2010; Letkewicz et al. 2013; Davenport and Parker 2015a; Richardson 2016; Davenport et al. 2019). Thus, this gap between previously identified sensitivities of supercell longevity (among other storm attributes) with environment characteristics and recent work regarding the influence of environmental variability on severe convection would benefit from additional investigation. Making use of high resolution environmental data throughout the lifetime of a supercell is one approach that could lead to better understanding of storm-environment interactions.

To further explore how supercell characteristics such as longevity and severe weather production are sensitive to environmental variability, the extent of such changes observed over the lifetime of supercell thunderstorms needs to be quantified. Bunkers et al. (2006a,b) focused on connecting broadly representative environments with supercell longevity and characteristics such as overall production of severe weather. In the present study, emphasis is placed on leveraging model analysis data with significantly greater spatiotemporal availability to identify the relationship between supercell characteristics and changes in the environment throughout the lifetime of the storm. Specifically, the goal of this paper is to measure the changes present in the near-inflow environment (via a number of common forecasting parameters) throughout the lifetime of long-lived supercells using a Lagrangian, storm-following framework; changes will then be evaluated with respect to metrics such as longevity and severe weather production. The long-term goal of this work is to enhance short-term forecasts of supercells and improve our understanding of their governing processes.

The paper is outlined as follows. Section 2 describes the set of long-lived supercells used for this study, along with the model soundings and parameters used to characterize their near-inflow environments. Evaluation of the climatological characteristics and environmental variability is presented in section 3, including how the results change for different subsets of supercells. Last, key findings are summarized in section 4, along with suggestions for future research.

\section{Data and methods}

A large number of observed supercells were identified for this study using a process similar to the one described in
Bunkers et al. (2006a), involving a manual examination of archived WSR-88D level II radar data to diagnose the presence of supercells. Using radar data between 2002 and 2017, candidate cases were initially selected based on the apparent persistence of several supercellular reflectivity characteristics such as hook echoes, strong reflectivity gradients on the inflow side of the storm, and deviant motion. Each candidate storm was further interrogated using WSR-88D velocity data at a variety of low and midlevel scan angles (at least the lowest three elevation tilts, including the base scan) to confirm the presence of a mesocyclone (rotational velocity of at least $15 \mathrm{~m} \mathrm{~s}^{-1}$; e.g., Andra 1997). To meet the long-lived supercell duration criteria, a mesocyclone needed to persist for at least $4 \mathrm{~h}$. Short periods ( $15 \mathrm{~min}$ or less) of missing velocity data (either due to limited radar coverage or simply being unavailable) or weaker rotation due to cyclic or episodic storm evolution were acceptable to meet the persistence definition. Cases without available velocity data for more than $15 \mathrm{~min}$, or cases with rotation continuously below mesocyclone strength for more than $15 \mathrm{~min}$ (resulting in a total supercellular period less than $4 \mathrm{~h}$ ) were discarded. Information regarding total storm lifetime was also recorded; storm initiation was defined as the time at which base reflectivity first exceeded $40 \mathrm{dBZ}$, while storm dissipation was defined as the time as which base reflectivity decreased below $40 \mathrm{~dB} Z$.

In contrast to Bunkers et al. (2006a), geographic focus was placed on the Great Plains region to quantify environmental variability away from the complicating effects of terrain. Furthermore, this study only included supercell cases that were relatively isolated (i.e., separated by at least one storm diameter from other storm cells) throughout their lifetime; this limitation was included so as to remove the effects of storm interactions on supercell evolution (e.g., Hastings and Richardson 2016) and make connections between modelestimated environmental parameter changes and storm response. While such interactions are fairly common and contribute to observed environmental variability in the storm environment with attendant impacts to supercell evolution, this exploration is left for future work. Altogether, 147 cases of long-lived, isolated supercells were identified for this study. As in Bunkers et al. (2006a), nearly all supercells in the dataset were right-movers; only two long-lived supercells were left-movers.

Next, each supercell thunderstorm was tracked over its entire lifetime to facilitate generation of model-based near-storm environmental profiles using the 0-h analysis from either the Rapid Update Cycle (RUC) or the Rapid Refresh (RAP) model (Benjamin et al. 2004, 2016). Note that model choice and grid spacing varied throughout the study period. Between May 2002 and the end of 2004, cases used RUC data containing 40-km horizontal grid spacing, while between 2005 and March 2007, the 20-km RUC data were used. Cases between April 2007 and April 2012 utilized the 13-km RUC data. Starting in May 2012, RAP model analyses were used, containing 13-km horizontal grid spacing. While there are inherent biases and errors with model data (e.g., Thompson et al. 2003; Coniglio 2012; Cohen et al. 2015), the significant spatiotemporal frequency of model data availability in comparison to observed 
rawinsondes (collected twice a day at select locations across the country) is strongly preferred to quantify environmental changes for the purposes of this study.

A latitude and longitude model grid point was chosen at each hour to represent the near-inflow environment over the lifetime of each supercell (see data availability statement). The process, described below, was inherently subjective and not without limitations. Selecting a "representative" proximity sounding implicitly recognizes that there is spatial and temporal variability in an environment (e.g., due to diurnal effects, surface boundaries, etc.) that cannot be sufficiently characterized in a single profile (see discussions in Brooks et al. 1994; Markowski et al. 1998b; Potvin et al. 2010, among others). While some studies have embraced an objective approach to ensure consistency in selection of the near-inflow point (e.g., Anderson-Frey et al. 2016; Coffer et al. 2020), the present study uses a more subjective procedure to better reflect the likely airmass contributing to the supercell's structure, intensity, and evolution. This unavoidably neglects the instantaneous effects of spatial variability due to boundaries (e.g., circulations and associated local enhancements in helicity, moisture, etc.) that are recognized to be influential (e.g., Markowski et al. 1998a; Boustead et al. 2013; Magee and Davenport 2020), as well as storm-induced variability in the inflow environment (e.g., Frame and Markowski 2013; Parker 2014; Nowotarski and Markowski 2016). However, a storm-relative framework that assumes a storm encounters a new environment at some rate has been shown to reasonably reproduce storm morphology (e.g., Davenport and Parker 2015a; Davenport et al. 2019), and is thus used herein as a means to capture connections between the environment and storm response.

The initial sounding time was chosen such that the closest model analysis time before initiation occurred was used, unless initiation occurred within $15 \mathrm{~min}$ of the next hour. For example, an initiation time of 1830 UTC would use the 1800 UTC analysis, while an initiation time of 1850 UTC would use the 1900 UTC analysis, as it was more likely to be representative. For initiation times close to the 15-min threshold (e.g., 1840 UTC), both the preceding and subsequent model analysis times were examined to qualitatively determine which was the most representative, focusing on relevant surface features and comparison to available observations. The latitude and longitude of the initial sounding model grid point was selected by either estimating the approximate location of storm formation (for analysis times chosen before initiation), or an appropriate near-inflow point (for analysis times chosen after initiation during early stages of storm lifetime), as described next.

Once the supercell formed, subsequent environment points at each hour were chosen by the following process. First, the location of the storm updraft was identified using a radar volume scan closest to the model analysis time. Next, based on estimated storm motion over the previous $15-\mathrm{min}$ period, a storm-relative upwind point approximately $30 \mathrm{~km}$ from the updraft was chosen (i.e., "near-inflow" as in Parker 2014), though this distance was unavoidably sensitive to the date of the case (i.e., changes in RUC/RAP horizontal grid spacing) and the reasonableness of the analysis with respect to available observations. Each profile was manually examined to determine representativeness, largely focusing on comparisons to the closest surface observation and available rawinsondes. Additionally, a visual inspection was conducted to ensure there was no convective contamination, typically apparent as regions of high relative humidity and weak lapse rates. Given the emphasis on isolated supercells in the present dataset, contamination largely occurred if the selected point was too close to the storm of interest due to available resolution of the analysis. Thus, larger distances were occasionally needed to achieve a sufficiently representative environment. While potentially problematic given observed spatial differences in the vicinity of supercells (e.g., Potvin et al. 2010; Parker 2014; Wade et al. 2018), to the extent possible, the same storm-relative distance was used throughout the near-inflow point selection process for each storm to ensure consistency.

Following generation of these near-inflow environment profiles, numerous parameters were computed for each sounding. Convective available potential energy (CAPE) and convective inhibition (CIN) using surface-based (SB), mixed-layer (ML, using the lowest $1000 \mathrm{~m}$ ), and most-unstable (MU) parcels were calculated using the virtual temperature correction (Doswell and Rasmussen 1994). Low-level and deep-layer bulk wind shear was computed over the 0-1-, 0-3-, 0-6-, and 0-8-km layers (as in Bunkers et al. 2006a), in addition to maximum bulk shear (defined as the maximum shear vector magnitude between 0-1 and $6-10 \mathrm{~km})$. Storm-relative helicity (SRH) within the $0-1$ - and 0-3-km layers was calculated using Bunkers-estimated storm motion (Bunkers et al. 2000). Effective bulk shear and SRH were also computed, along with the depth of the effective inflow layer; effective layer parameters are defined as in Thompson et al. (2007), limited to the layer in which CAPE $\geq 100 \mathrm{~J} \mathrm{~kg}^{-1}$ and $\mathrm{CIN} \geq-250 \mathrm{~J} \mathrm{~kg}^{-1}$. The supercell composite parameter (SCP), as formulated in Gropp and Davenport (2018), was computed to quantify the extent to which environments supported supercells (Thompson et al. 2003). Other parameters relevant to long-lived supercells identified in Bunkers et al. (2006a) were also included: ML lifting condensation level (MLLCL) and ML level of free convection (MLLFC).

Last, tornado and severe storm reports were gathered from Storm Data for each supercell. There are several welldocumented issues with the database, including underreporting of weak tornadoes (E/F0 and E/F1), a secular/nonmeteorological increase in reports over time, and human error in reporting (e.g., Doswell and Burgess 1988; Weiss et al. 2002; Brooks et al. 2003; Edwards et al. 2018). Even so, valuable information regarding broad trends and relative frequency of severe weather production (including severe and significant severe) ${ }^{1}$ was gathered based on the presence and timing of storm reports, as opposed to focusing on the exact number of reports produced. Additionally, as a result of likely overestimation of estimated severe wind gusts (Edwards et al. 2018), this study only included measured gusts in the analysis.

\footnotetext{
${ }^{1}$ Defined as $\mathrm{F} / \mathrm{EF} 2-\mathrm{F} / \mathrm{EF} 5$, hail diameter $\geq 5.1 \mathrm{~cm}$ (2.0 in.), and/or wind gusts $\geq 33.5 \mathrm{~m} \mathrm{~s}^{-1}$ (65 kt; see Hales 1988).
} 


\section{Results}

\section{a. Climatological characteristics}

To begin, an overview of the geographic distribution of supercells analyzed in this study is shown in Fig. 1. Broadly, the distribution of storm tracks is similar to those within the southcentral and north-central United States as shown in Bunkers et al. (2006a); long-lived supercells occur most frequently in the southern Great Plains, and generally exhibit motion toward the northeast, east, or southeast. As with the Bunkers et al. (2006a) dataset, there are a few anomalous tracks, such as a few supercells moving straight southward, but the majority display typical supercellular motion tendencies (i.e., deviant motion).

Long-lived supercells occur most frequently in late spring and early summer (Fig. 2a), consistent with severe weather climatology and prior examination of long-lived supercell events (e.g., Bunkers et al. 2006a; Smith et al. 2012; Reames 2017; Li et al. 2020). The strong influence of diurnal heating is clear, with over $80 \%$ of cases existing at either 0000 or 0100 UTC (Fig. 2b). This diurnal signal is further underscored when examining the distribution of times for supercell initiation and dissipation. A plurality of cases initiated between 2100 and 2200 UTC, and then dissipated between 0200 and 0400 UTC (Fig. 3a). Accordingly, the average lifetime of a long-lived supercell was $5.5 \mathrm{~h}$. However, the distribution of supercell lifetimes is strongly skewed; the bulk of cases exhibited lifetimes of less than $5 \mathrm{~h}$, with an exponential decrease in frequency for longer lifetimes (Fig. 3b).

Severe weather production for the supercells in this dataset is similar to other studies in which the majority of supercells comprising those studies were associated with severe reports (e.g., Burgess and Lemon 1991; Bunkers et al. 2006a; Smith et al. 2012). The most common severe hazard with supercells is hail, followed by tornadoes and then measured severe wind gusts (Table 1). Focusing on the most significant severe weather produced in each report type (i.e., identifying the highest tornado rating, largest hail, and/or strongest wind gust) for each case overall, as well as the most significant produced each hour for a storm, Fig. 4 illustrates a spectrum of severe weather production. Tornado reports exhibit the expected distribution weighted toward weaker tornadoes (e.g., Brooks and Doswell 2001), along with notable diurnal signals; tornadoes tend to occur around 00000100 UTC, consistent with the supercell diurnal peak (cf. Fig. 2b), as well as a peak in significant tornadoes after 0200 UTC, likely related to the climatological increase in low-level shear as a result of the nocturnal low-level jet (e.g., Coffer and Parker 2015; Figs. 4a,b). The distribution of hail reports in Fig. 4c is strongly clustered in the 2.5-3in. diameter bin, which is likely a result of reports tending to be provided based on reference objects (e.g., Allen et al. 2020). However, the timing of the reports exhibits a clear diurnal trend, with the distribution of different hail sizes remaining relatively constant throughout the day, though there is a slight preference for more significant hail between 2300 and 0100 UTC (Fig. 4d). Measured severe wind gusts, like tornadoes, are skewed toward the weakest intensities (Fig. 4e). The timing of severe gusts is largely

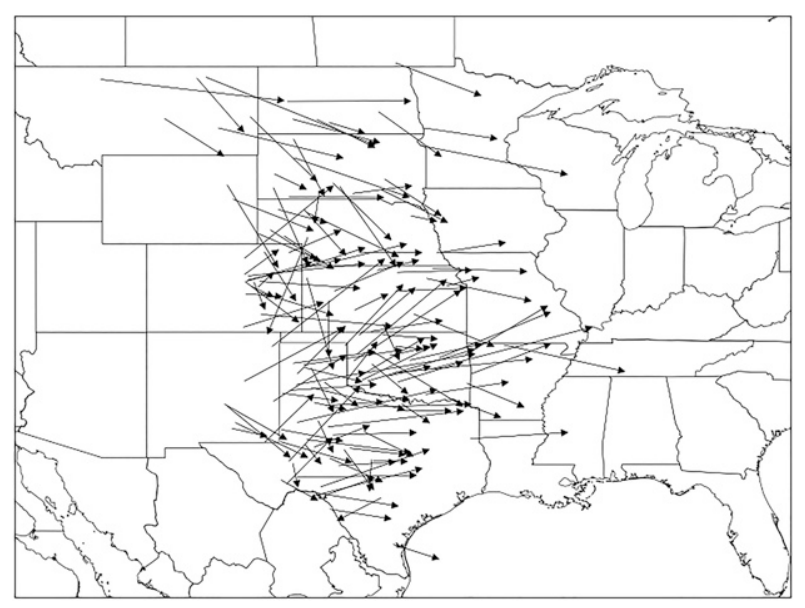

FIG. 1. Tracks of the 147 long-lived supercell cases examined in this study. The tail of each arrow indicates the starting position, while the tip of the arrow indicates where supercell demise occurred.

diurnal in nature, though the occurrence of significantly severe gusts varies throughout the day (Fig. 4f).

\section{b. Environmental evolution}

\section{1) DIURNAL}

Given the strong relationship between long-lived supercell initiation or dissipation, severe weather production, and the diurnal cycle, a similar signal exists in the nearstorm environments of these events. The hourly distribution of buoyancy and inhibition demonstrates that MLCAPE (MLCIN) is typically maximized (minimized) in the late afternoon hours, which is also when initiation tends to occur, with the reverse being observed in the evening after sunset when dissipation is common (Fig. 5; cf. Fig. 3a). Similar patterns are evident in SB and MU buoyancy and inhibition parameters (not shown).

The diurnal cycle not only influences buoyancy and inhibition, but also the low-level wind profile and related parameters; diurnal patterns in shear and SRH are well-established and commonly observed in convective environments in the Great Plains (e.g., Maddox 1993; Markowski et al. 1998b; Kis and Straka 2010; Mead and Thompson 2011; Parker 2014; Reames 2017; Gropp and Davenport 2018). In the present dataset, a steady and notable increase in $0-1-\mathrm{km}$ bulk shear is evident after 0000 UTC (Fig. 6a), coincident with the climatological development of the nocturnal low-level jet (e.g., Kerr and Darkow 1996). Relatedly, 0-1-km SRH also strongly increases after 0000 UTC (Fig. 6b). Within the $0-3-\mathrm{km}$ layer, the post0000 UTC increase is still apparent in SRH, but not as distinct in bulk shear (not shown).

The persistence of some long-lived supercells into the evening hours (cf. Fig. 3), even with accompanying decreases in buoyancy and enhanced inhibition (Fig. 5), suggests that effectivelayer parameters should also be examined to provide a more meaningful measure of the environment influencing the storm 
a)

Monthly Frequency

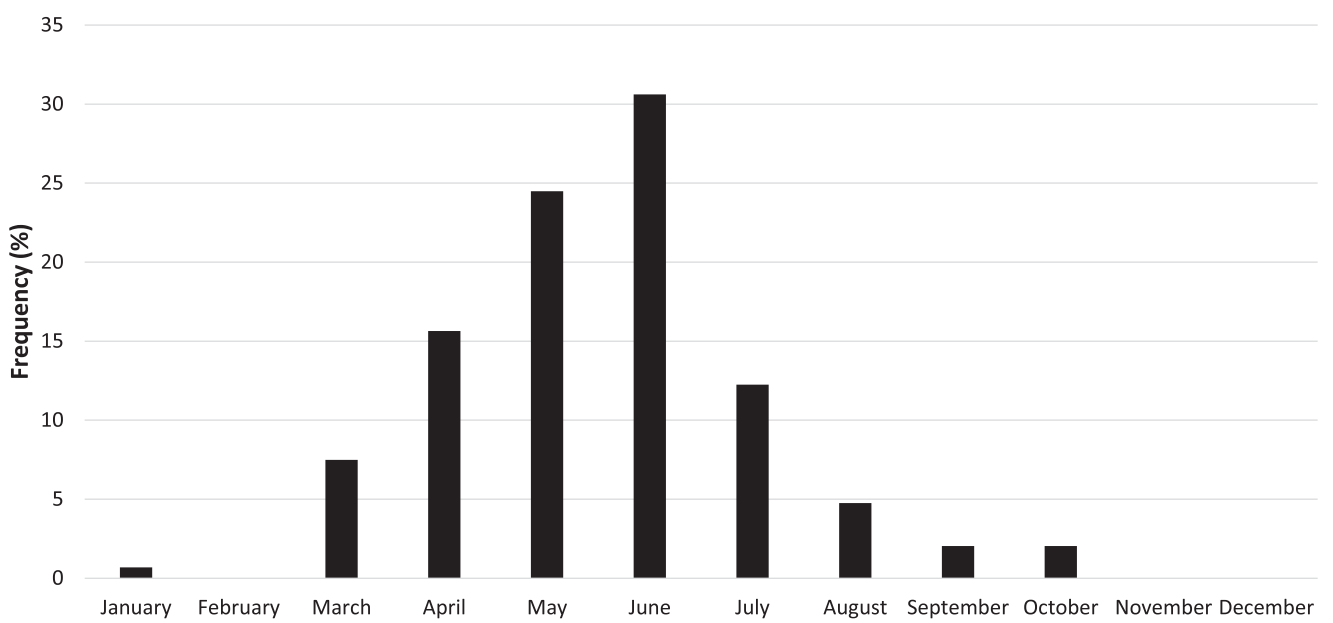

b)

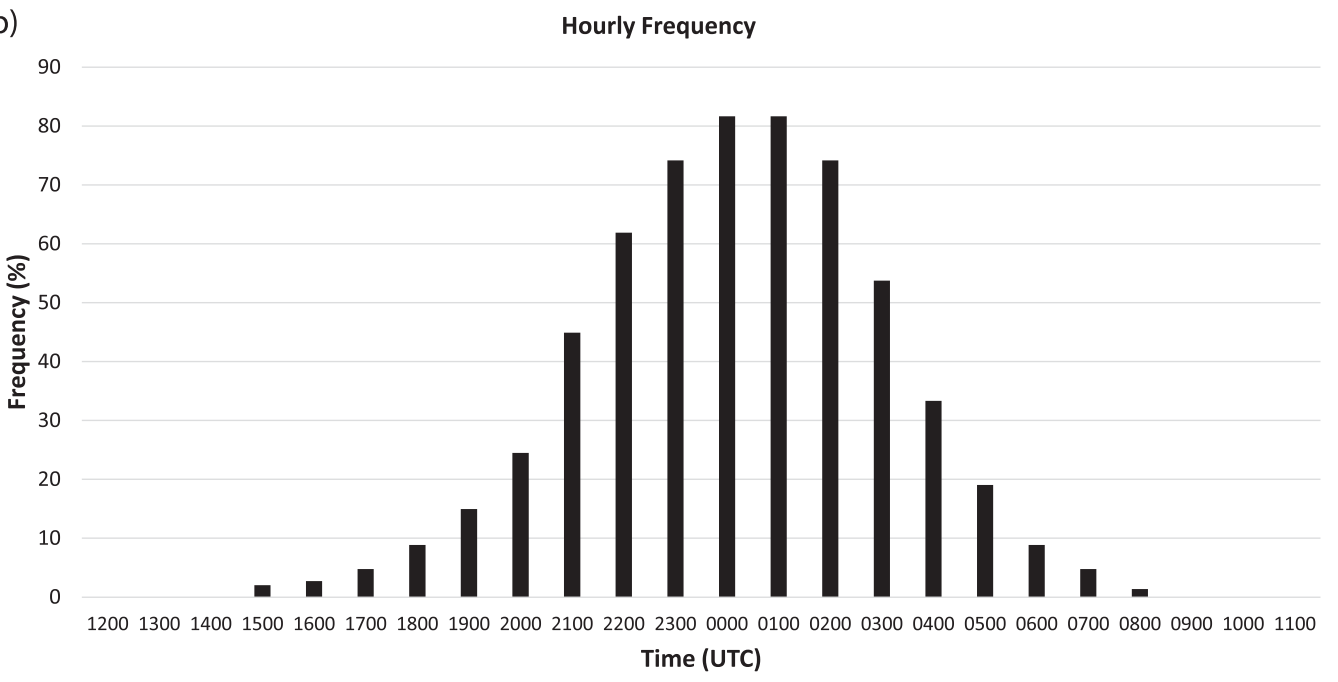

FIG. 2. Frequency of long-lived supercells binned by (a) month of occurrence and (b) hourly occurrence. Note that in (b), storms can be present in more than one bin, depending on how long they met the criteria for being a supercell (i.e., when a mesocyclone was present).

updraft (e.g., Thompson et al. 2007; Gropp and Davenport 2018). While the effective-layer shear does not strongly vary throughout the day (Fig. 7a), there is a notable nocturnal increase in effective SRH, consistent with the fixed-layer SRH parameters (Fig. 7b; cf. Fig. 6). A combination of buoyancy, inhibition, and effective layer parameters via SCP demonstrates relative consistency in the interquartile range throughout the day, though median values exhibit more variation (Fig. 8).

\section{2) MATURITY-RELATIVE}

It is clear that the diurnal cycle has a strong influence on the initiation and dissipation of long-lived supercells (Fig. 3a), as well as their local environments (Figs. 5-8). However, to understand how supercells interact with their environments to result in maintenance, weakening, or strengthening of the rotating updraft, the near-inflow environments observed at different stages of storm maturity need to be assessed. Accordingly, each long-lived supercell was assigned a sounding to represent the initial, mature, and dissipation stages of the storm. The initial environment for each storm was simply the first sounding generated for a storm, representative of either the pre-convective or early stage environment that the supercell formed in (see earlier discussion in section 2). For the mature stage, the hour in which the storm reached peak, yet quasi-steady intensity (i.e., not rapidly increasing or decreasing in magnitude) occurred was identified; this classification was based on timing of peak magnitude of rotational velocity. Corresponding reflectivity data were also examined to further ensure that supercellular reflectivity characteristics, such as a hook echo or bounded weak echo regions, were quasi-steady and not rapidly evolving or 
a) Time of Supercell Initiation and Dissipation

25

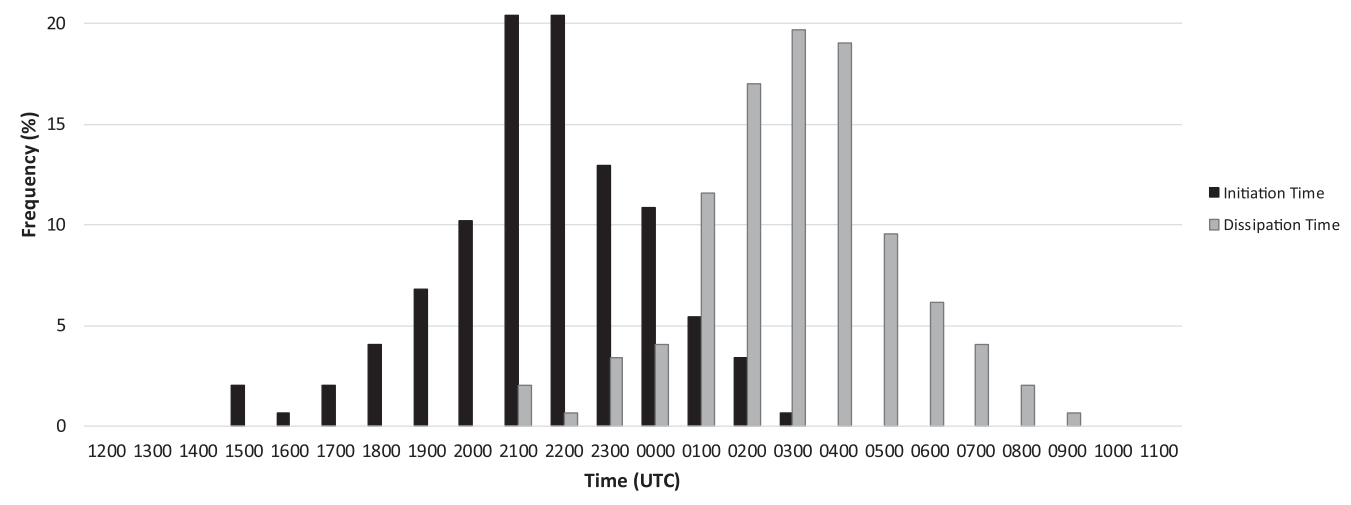

b)

Lifetime Frequency

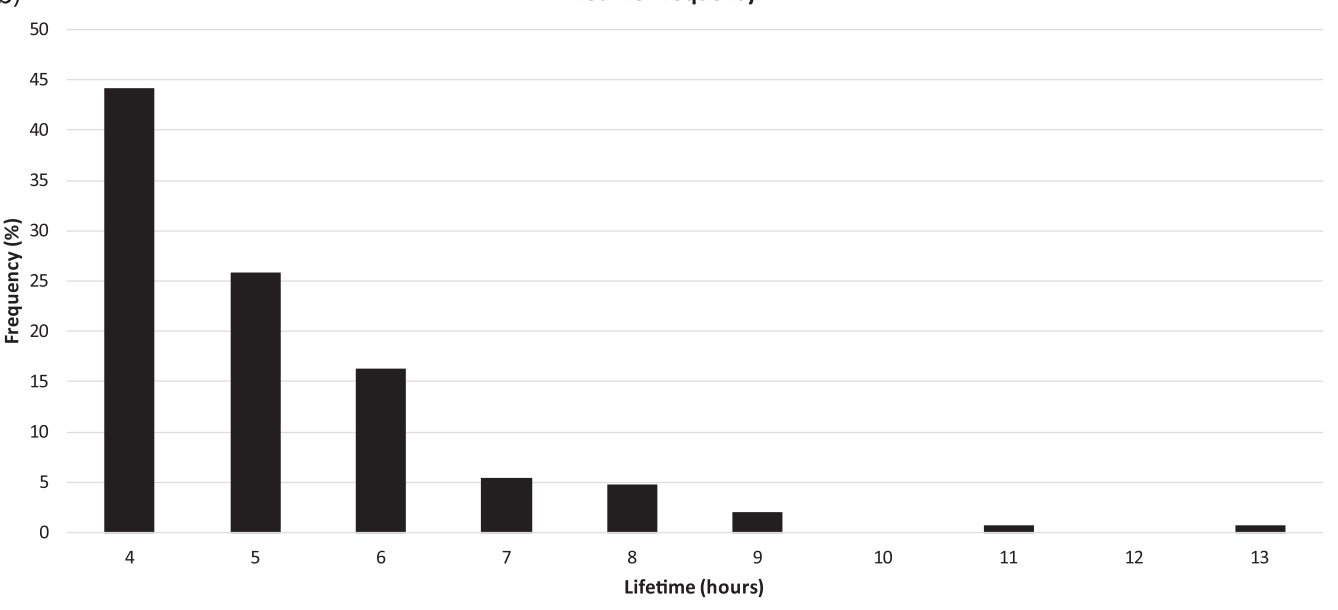

FIG. 3. Frequency of long-lived supercells binned by (a) time of initiation or dissipation and (b) overall lifetime. Note that frequencies are related to when the supercell's mesocyclone was observed, rather than total storm lifetime.

cycling. The dissipation environment was assigned as the final sounding generated for each supercell.

Broadly, thermodynamic buoyancy tends to steadily decrease over the lifetime of this supercell sample, particularly for SB and MU parcels, while ML exhibits more notable decreases between the mature and dissipation stages (Figs. 9a-c). Trends in the magnitude of convective inhibition over the lifetime of a supercell are somewhat dependent on the parcel of interest. SBCIN generally strengthens over time, particularly at the dissipation stage, though the distribution narrows in the -100 to $-200 \mathrm{~J} \mathrm{~kg}^{-1}$ range compared to the initial stage (Fig. 9d). MLCIN, in contrast, exhibits a steady widening of the distribution of values over time, along with an increase in the range of values (Fig. 9e). MUCIN in Fig. 9f exhibits a similar trend as SBCIN. Despite some variability and dependence on parcel selection, the patterns described above are consistent with the strong diurnal signal and typical initiation and dissipation times (Figs. 3a, 5), resulting in decreases in buoyancy and increases in inhibition over time.

The evolution of various shear parameters differed, largely based on whether the shear was measured within the low levels or over a deeper layer. For example, 0-1-km bulk shear notably increased over time, with the largest values observed during the dissipation stage (Fig. 10a). Over a slightly deeper layer, trends in the $0-3-\mathrm{km}$ and effective layer shear over time

TABLE 1. Frequency of severe weather production (relative to all cases) binned by report type. Note that the wind report category only includes measured severe wind gusts.

\begin{tabular}{lcc}
\hline \hline Report type & Percent occurrence (\%) & Percent significant (\%) \\
\hline Tornado & 59.2 & 22.4 \\
Hail & 99.3 & 70.7 \\
Wind & 40.8 & 11.6 \\
\hline
\end{tabular}



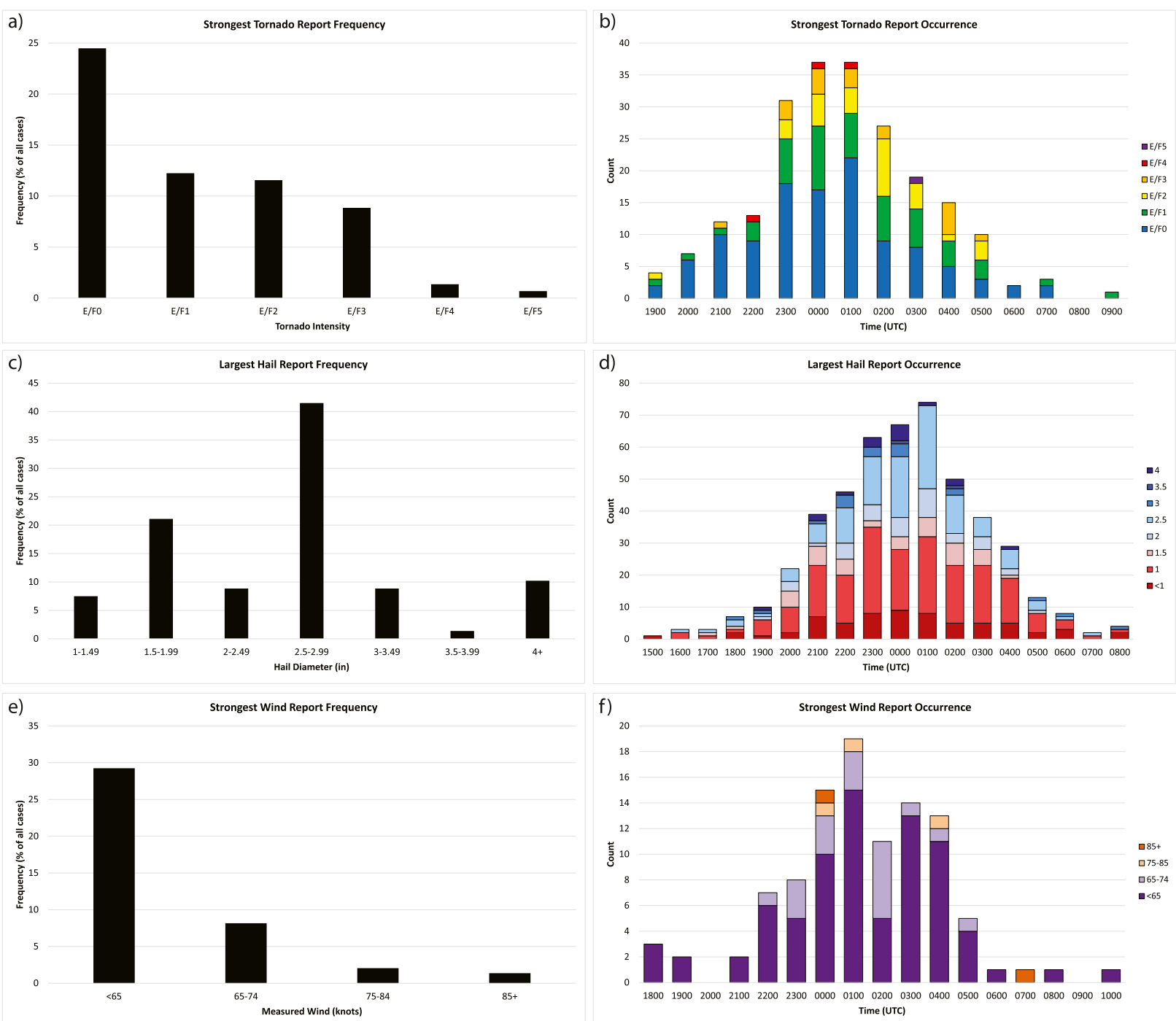

FIG. 4. Distributions of the most significant severe report in each category per case are shown, illustrating (a) the most damaging tornado, (c) the largest hailstone, and (e) the strongest wind gust for each case. (b),(d),(f) Distributions of the most significant severe report in each category within each hour throughout a supercell's lifetime.

displayed a slight increase in values between the initial and mature stages, followed by a decrease in values at the dissipation stage (Figs. 10b,e). Deep-layer bulk shear within 0-6 and 0-8 km, as well as max bulk shear all exhibited slight weakening of shear on the order of a few meters per second between the mature and dissipation stage. However, these slight decreases are not likely to be physically impactful, and could be within observational error.

The storm-relative helicity parameters all demonstrated increases over time (Figs. $10 \mathrm{~g}-\mathrm{i}$ ). The trend is most dramatic for 0-1-km SRH, consistent with the diurnal low-level jet signal (Fig. 6) and the timing of dissipation of many supercells during the overnight hours (Fig. 3a). Smaller increases over the lifetime of the supercell cases were present in both $0-3-\mathrm{km}$ and effective SRH (Figs. 10h,i). Given these relatively smaller changes in effective-layer shear and SRH, the notable decrease in SCP at the dissipation stage reflects reductions in buoyancy and larger inhibition (Fig. 10j; cf. Figs. 9c,f). Furthermore, such decreases in SCP reflect an environment less favorable for supercells (e.g., Thompson et al. 2003; Gropp and Davenport 2018).

\section{c. Subset comparisons}

The above trends are revealing of the complexity of the evolution in the near-storm environment, with the diurnal cycle being a key signal. However, it may be instructive to determine if there are other patterns of importance. Accordingly, two subsets of cases are analyzed in more detail below.

\section{1) MARginAlly VERSUS VERY LONG-LIVED SUPERCELLS}

Recall that the distribution of supercell lifetimes was skewed toward lengths less than $5 \mathrm{~h}$ for this sample of long-lived 
a)

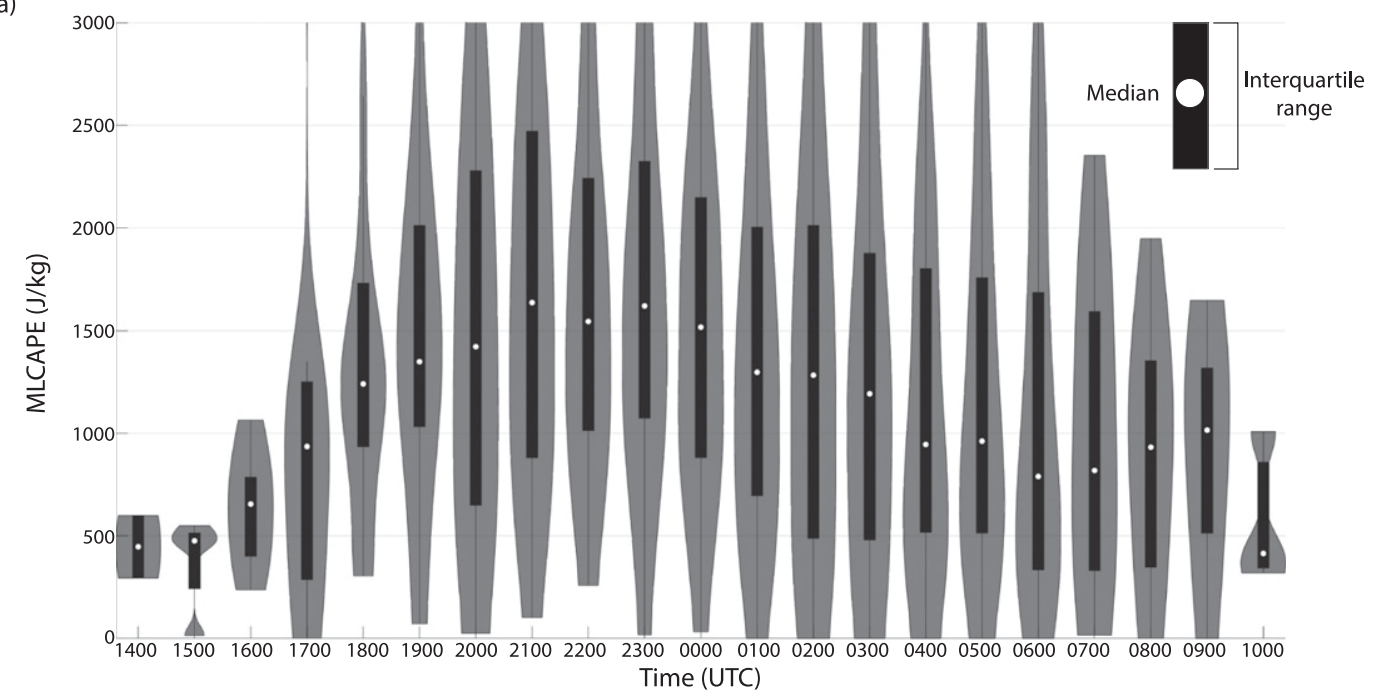

b)

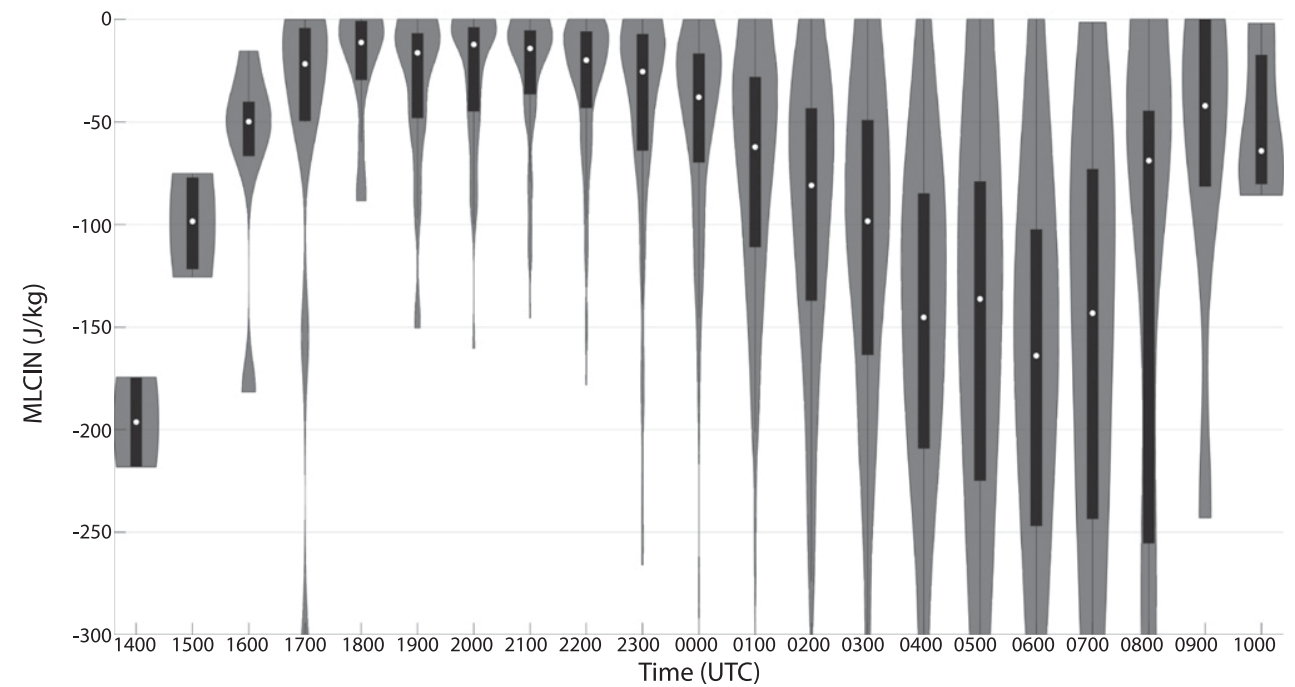

FIG. 5. Violin plots of the distribution of (a) MLCAPE and (b) MLCIN values at each hour of the day in which long-lived supercells were present (including their pre- and post-mesoscylone environments). The violin plot is overlayed by a box-and-whiskers plot containing the median (white circle), interquartile range (thick black bar), minimum and maximum values (thin black bars), and kernel density estimate (gray fill). Where the plot is wide (narrow), there is a greater (smaller) probability that the raw data contains that value.

supercells (Fig. 3b). Given that there are a number of significant environmental differences between short-lived $(\leq 2 \mathrm{~h})$ and longlived ( $\geq 4 \mathrm{~h}$ ) supercells (Bunkers et al. 2006b), it may be useful to determine what environmental factors differ between marginally long-lived (defined here as $\geq 4$ but $<5 \mathrm{~h}$ ) and very long-lived $(\geq 7 \mathrm{~h})^{2}$ supercells. The present dataset contains 65 marginally long-lived supercells and 20 very long-lived supercells.

\footnotetext{
${ }^{2}$ The very long-lived threshold was chosen so as to examine the more extreme portion of the supercell lifetime distribution while still having a sufficient number of cases to compare to the marginally long-lived subset.
}

The first indication of environmental differences between marginally and very long-lived supercells is suggested by the variations in monthly frequency (Fig. 11a). While both categories of long-lived supercells occur throughout the spring and summer months, marginally long-lived supercell occurrence peaks in May and June, reflecting the distribution of all longlived supercells (Fig. 2a), and very long-lived supercells peak in April. There is a secondary peak in June as well, though its frequency is smaller relative to the frequency of marginally long-lived cases. The timing of supercell initiation illustrates that very long-lived supercells tend to form earlier in the day and survive longer into the overnight hours before dissipating (Fig. 11b). These patterns could be a result of stronger 


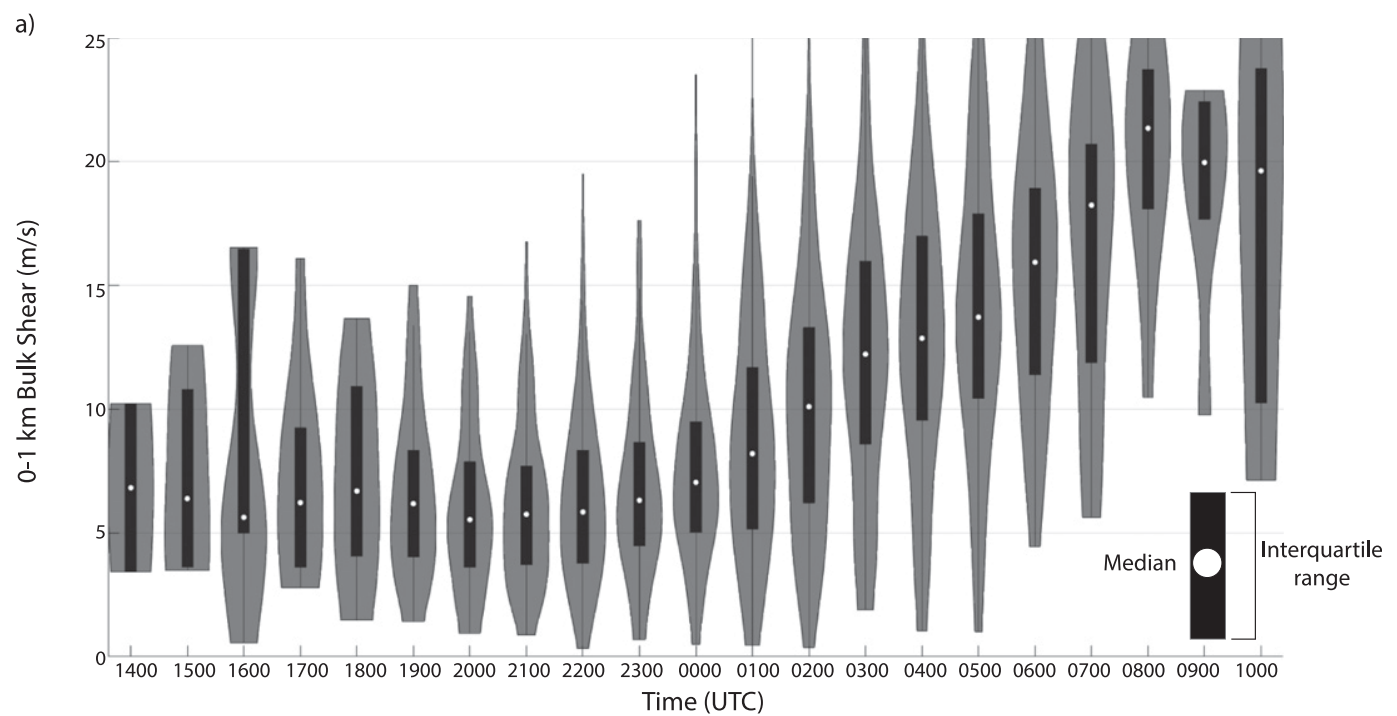

b)

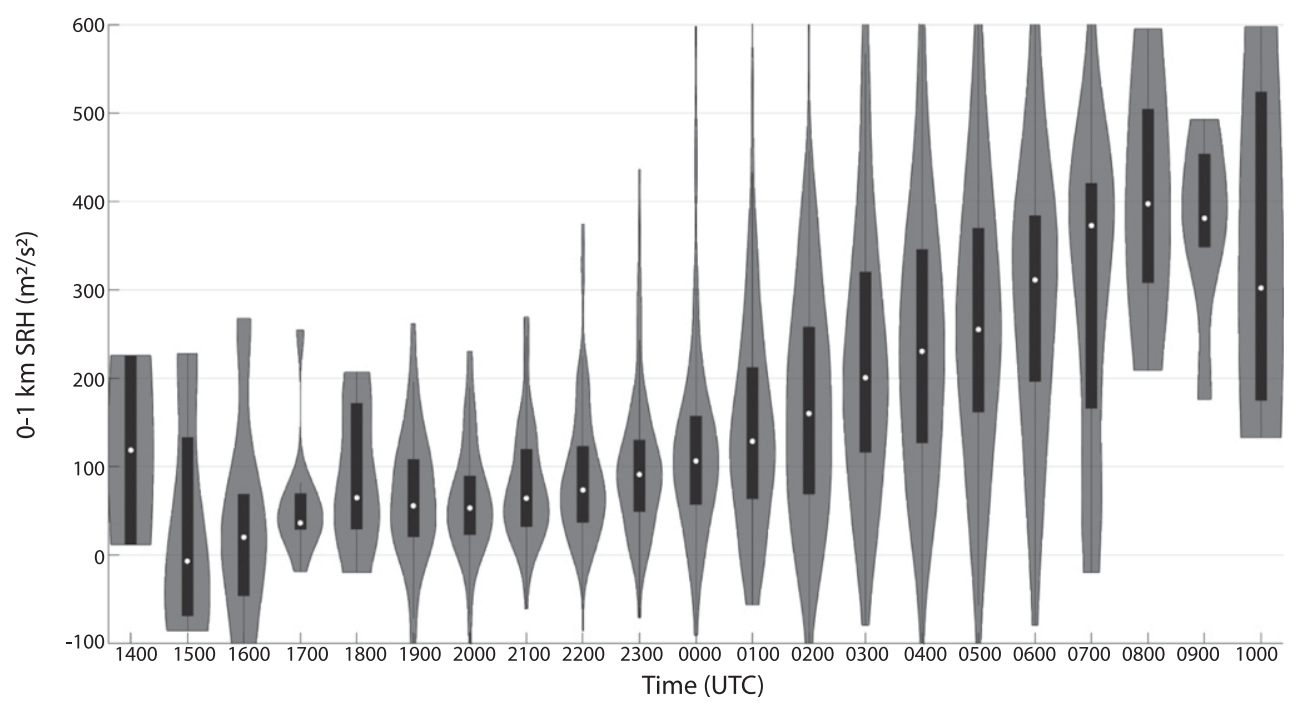

FIG. 6. As in Fig. 5, but for distributions of (a) 0-1-km bulk shear and (b) 0-1-km storm-relative helicity.

synoptic-scale forcing associated with springtime systems and a more intense midlatitude jet, but such explorations are outside the scope of this study and left for future work.

At the initial stage, the most prominent difference between marginally long-lived and very long-lived supercells lies in deep-layer bulk shear; indeed, the only statistically significantly different ${ }^{3}$ parameter was the $0-8-\mathrm{km}$ bulk wind shear. Very long-lived supercells form in environments with stronger deep-layer shear where the average and median value is 5$7 \mathrm{~m} \mathrm{~s}^{-1}$ higher for the longest-lived storms (Table 2; Fig. 12a). This finding is consistent with a higher frequency of very long-

\footnotetext{
${ }^{3}$ Statistical test based on the nonparametric KolmogorovSmirnov test (Wilks 2011), used as a result of the nonnormality of parameter distributions.
}

lived supercell occurrence in April (Fig. 11a) when the midlatitude jet tends to be stronger. Furthermore, Bunkers et al. (2006b) identified this same parameter as discriminatory between short- and long-lived supercells, which primarily linked stronger deep-layer shear to enhanced updraft strength and rotation, leading to extended storm longevity. Other bulk shear parameters also tended to exhibit higher initial values for very long-lived storms, but those differences were not statistically significant (Table 2; Figs. 12b,c).

Once supercells reach the mature stage, more substantial differences in the environments of the subgroups were present. In addition to much stronger $0-8-\mathrm{km}$ bulk shear, shear within $0-3-\mathrm{km}$ and effective layers were significantly larger for the very long-lived storms (Table 2; Figs. 12b,c). The significance of effective layer bulk shear is present in spite of the overall effective layer depths being similar (not shown). 


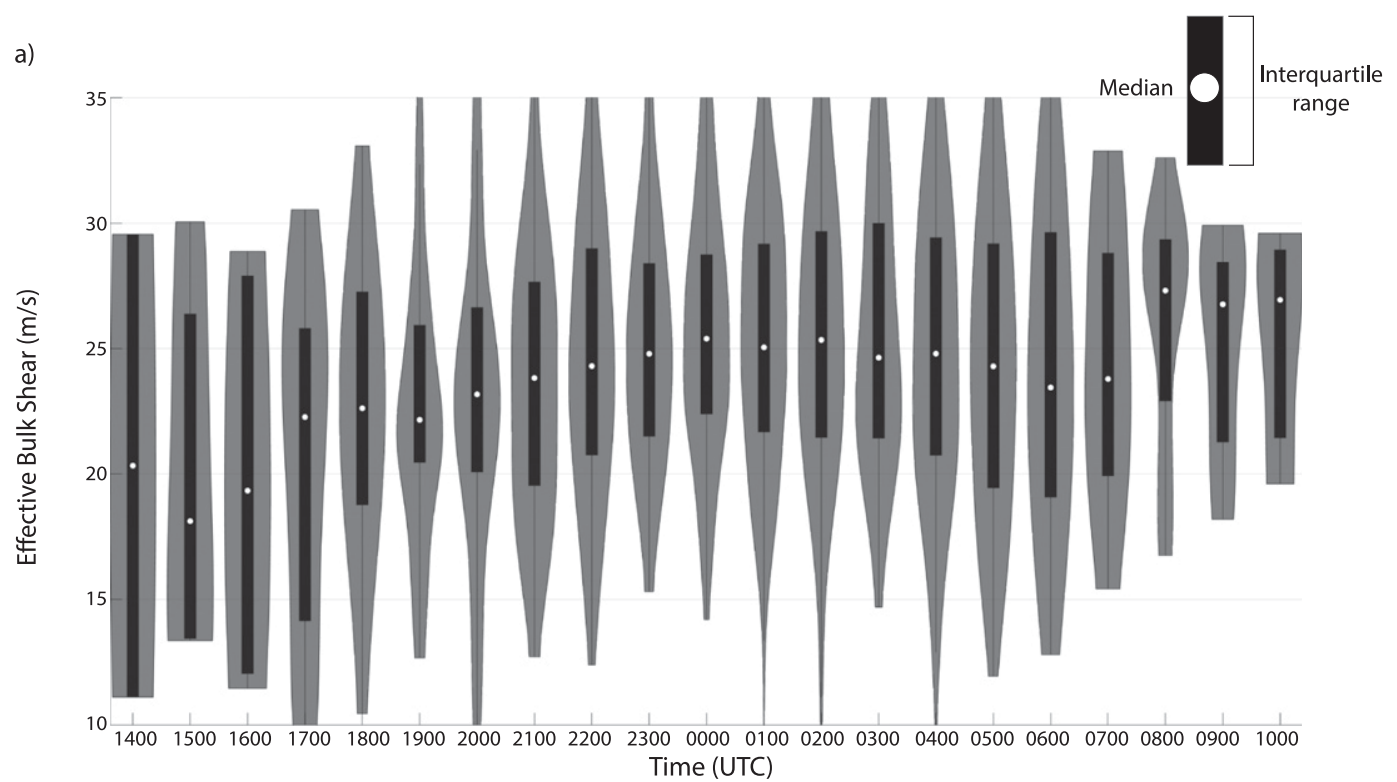

b)

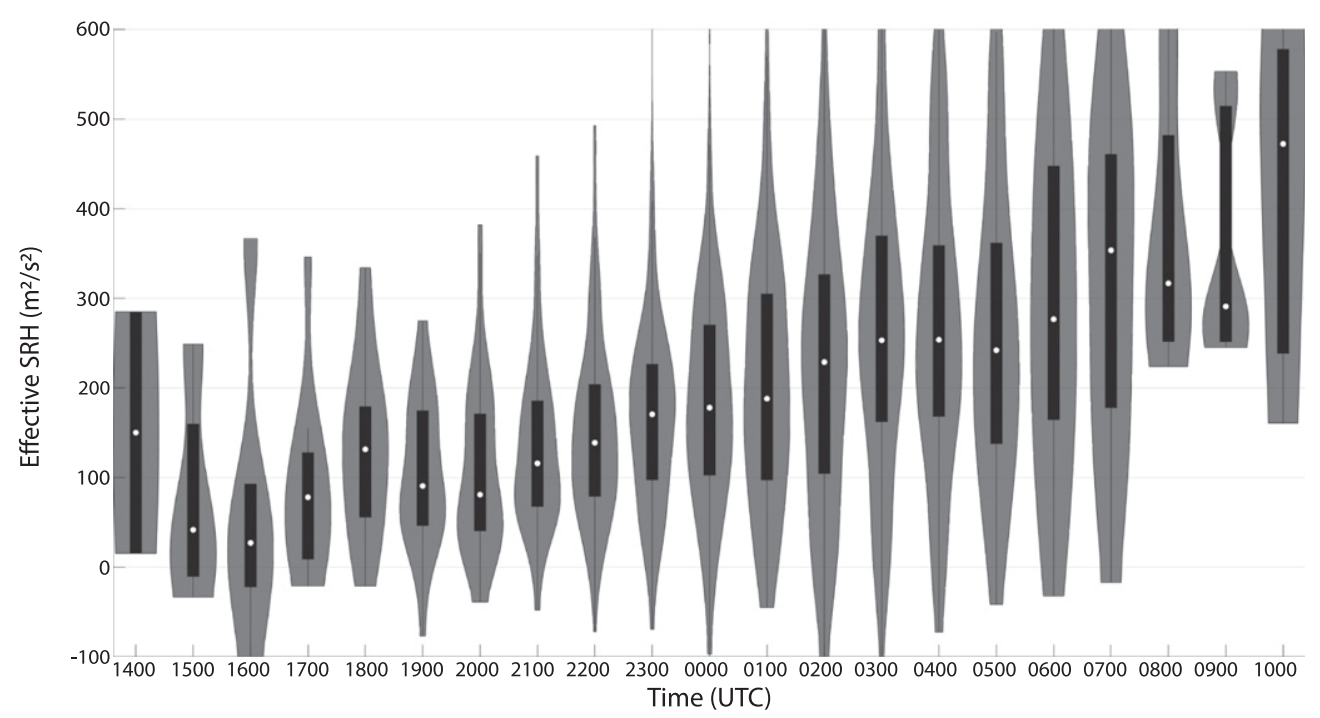

FIG. 7. As in Fig. 5, but for distributions of (a) effective bulk shear and (b) effective storm-relative helicity.

Additionally, storm-relative helicity within the $0-3-\mathrm{km}$ layer also became significantly stronger for the longestlived supercells, a notable evolution given the near-overlap of marginally long-lived and very long-lived distributions at the initial stage (Fig. 12d).

At the dissipation stage, there were no statistically significant differences between marginally long-lived and very long-lived supercells (Table 2). Bulk shear in the $0-3-\mathrm{km}, 0-8-\mathrm{km}$, and effective layers generally weakened for all supercells relative to their mature stage, though very long-lived storms continued to maintain somewhat higher values (Figs. 12a-c). The only parameter to demonstrate some strengthening (based on the median value) from the mature to dissipation stage was 0-3-km SRH; again, the very long-lived storms continued to experience higher (though not significantly so) values (Fig. 12d).
These continued increases in SRH are consistent with dissipation in the overnight hours when the low-level jet is likely present (Fig. 11b). Notably, in spite of very long-lived supercells dissipating later, there were no significant differences in buoyancy or inhibition (not shown).

On the whole, the amount of initial deep-layer shear in the 0-8-km layer appears to be important in setting the stage for supporting overall supercell longevity. As the storm matures, increases in 0-3-km shear and SRH, especially large increases, would potentially help support continued updraft rotation (e.g., Coffer and Parker 2015; Davenport et al. 2019) and extend the longevity of the supercell. Such changes in a stormrelative sense in the near-inflow environment appear to be key, as individual parameters at the initial or mature stages have weak correlations with storm longevity (not shown). However, 


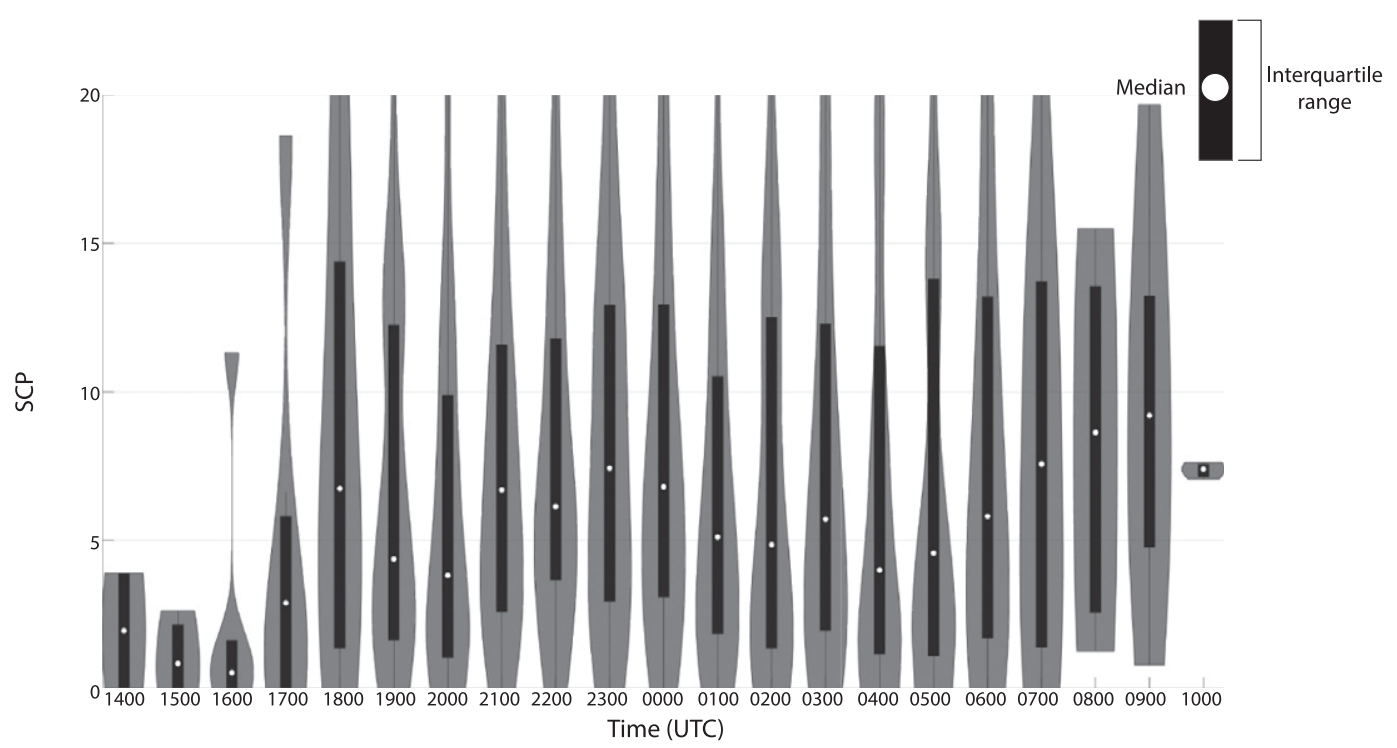

FIG. 8. As in Fig. 5, but for distributions of CIN-scaled SCP.

given the fairly small sample size of very long-lived supercells, it will be important to further explore these factors via controlled idealized experiments.

\section{2) BEFORE VERSUS AFTER SUNSET}

One of the most notable signals present in the present dataset of long-lived supercells is the influence of the diurnal cycle on initiation and dissipation (Fig. 3a) and the near-storm environment (e.g., Figs. 5-6). However, it may be worthwhile to identify the environmental controls on long-lived supercells that dissipate before sunset to parse any nondiurnal effects. For example, does the progression of the near-inflow environment evolution notably differ? Supercells were categorized based on the time that each storm was no longer considered a supercell (i.e., its rotational velocity weakened below $15 \mathrm{~m} \mathrm{~s}^{-1}$ ) relative to local sunset; those that lost rotation prior to sunset were binned as dissipating "before sunset," while those losing rotation postsunset were binned as dissipating "after sunset." A total of 31 supercells fell in the "before" sunset category, while 116 supercells were in the "after" category.

Figure 13 displays the maturity-relative evolution of select parameters for the before versus after sunset categories. While there are differences in parameter values between supercells that dissipate before versus after sunset, overall, the evolution
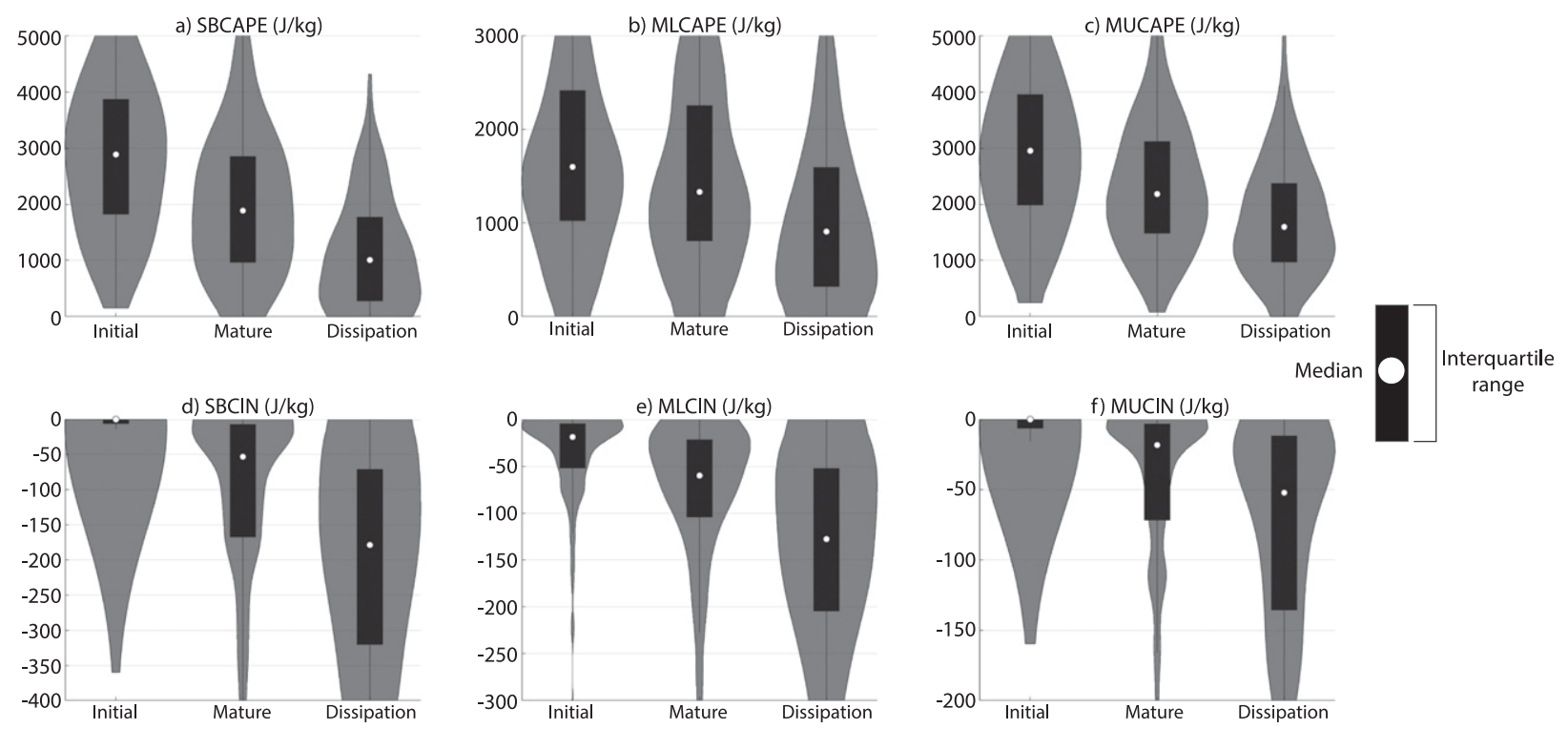

FIG. 9. Maturity-relative violin plots of select thermodynamic parameters. 

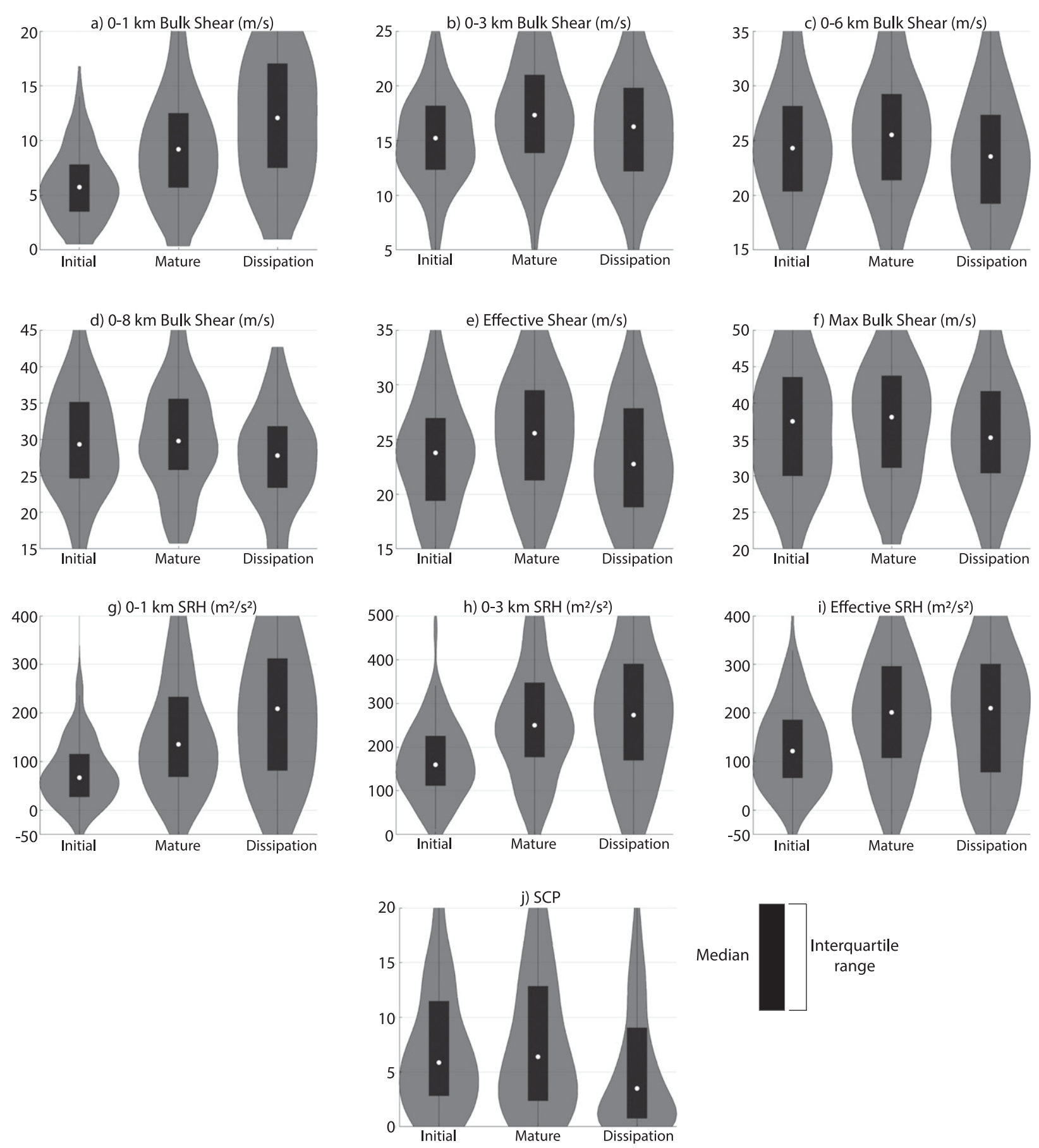

FIG. 10. As in Fig. 9, but for distributions of select bulk wind shear and SRH parameters and CIN-scaled SCP.

in distributions of many parameters from initial to mature to dissipation environments is largely similar. For example, MLCAPE and MLCIN display expected diurnal patterns of generally decreasing buoyancy and increasing inhibition over the lifetime of supercells, yet the trends start from different baselines; due to formation earlier in the day, supercells that dissipate before sunset generally contained much less buoyancy in their initial environment than those that dissipated after sunset (Figs. 13a,b). Similarly, shear in the 0-1$\mathrm{km}$ layer increases over time, though the magnitude of that shift is much more substantial for storms dissipating after sunset (Fig. 13c), likely due to the development of the nocturnal low-level jet. Deep-layer shear is somewhat different; supercells dissipating before sunset show little change in distribution values from initial to mature to dissipation environments, while supercells dissipating after sunset tend to exhibit 


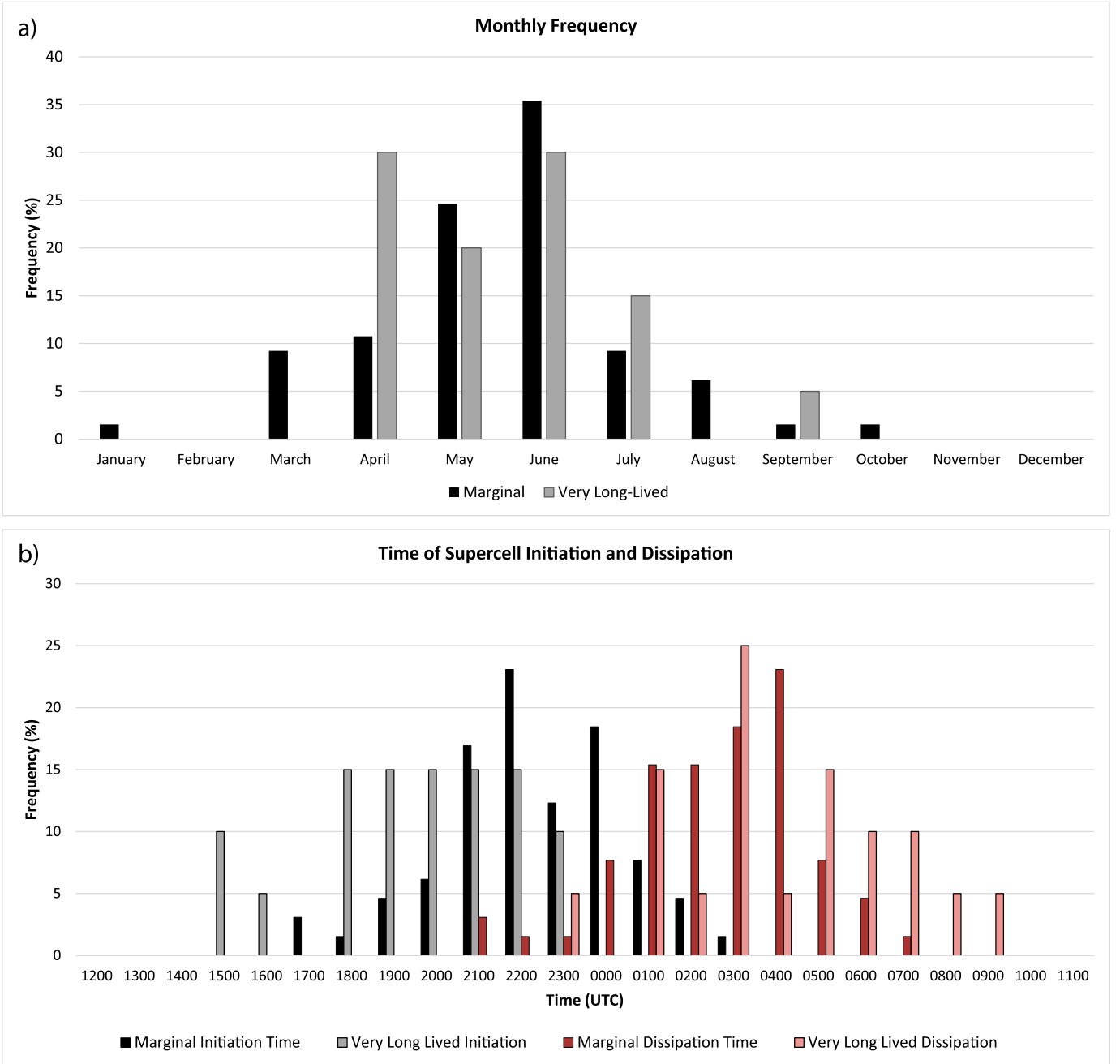

FIG. 11. Frequency of marginally long-lived ( $\geq 4$ but $<5 \mathrm{~h}$ ) vs very long-lived ( $\geq 7 \mathrm{~h}$ ) supercells binned by (a) month of occurrence and (b) time of initiation or dissipation.

slightly higher shear values that shift downward at the dissipation stage (Fig. 13d). Effective layer parameters displayed similar trends in distributions between the initial and dissipation stages, regardless of dissipation timing. Effective shear tended to peak at the mature stage in both subgroups, while effective SRH tended to be largest at the dissipation stage (Figs. 13e,f). Overall temporal trends in SCP were similar, with lowest values at the dissipation stage, though the magnitude of SCP was largely higher for supercells dissipating after sunset (Fig. 13g). Additional investigation of the impacts of diurnal versus nondiurnal effects will be left for future work.

\section{d. Severe weather production}

A key aspect of environmental variability that needs to be explored is the sensible impacts of such changes; in particular, the ability of environmental variations to influence severe weather production is briefly examined herein. To facilitate this examination, the time of each tornado, hail, and wind report was adjusted to the closest hour to be paired with a reasonably representative set of environmental parameters. For example, a report occurring at 2310 UTC was assigned to the 2300 UTC model environment, while a report occurring at 2350 UTC was assigned to the 0000 UTC model-generated environment profile. Additionally, the percent change in a parameter's value in the hour before a severe report occurred (relative to the previous environment) was computed. The percent change in a given parameter in the hour before a report is designed to provide information about the significance of the shift, given that parameter magnitudes can substantially vary between and within individual cases (e.g., Figs. 9-10). For further ease of interpretation, the data has been smoothed using a two-dimensional kernel density estimation using an optimization algorithm for the kernel bandwidth and a Gaussian kernel.

A wealth of prior research on tornadoes has demonstrated several key environmental components that contribute to their development in supercell thunderstorms, including buoyancy, high low-level humidity (usually quantified as low LCL heights), strong low-level vertical wind shear, and large SRH (e.g., 
TABLE 2. Average values of select parameters at the initial, mature, or dissipation stage, subset by supercells that were marginally $(\geq 4$ but $<5 \mathrm{~h}$ ) or very long-lived $(\geq 7 \mathrm{~h}$ ). The $p$ values listed are derived from the Kolmogorov-Smirnov nonparametric statistical test comparing the marginally long-lived vs very long-lived categories. Statistically significant values (at the $\alpha \leq 0.05$ level) are indicated in bold italics.

\begin{tabular}{|c|c|c|c|c|c|c|c|c|c|}
\hline \multirow[b]{2}{*}{ Parameter } & \multicolumn{3}{|c|}{ Initial } & \multicolumn{3}{|c|}{ Mature } & \multicolumn{3}{|c|}{ Dissipation } \\
\hline & Marginal & Very long & $p$ value & Marginal & Very long & $p$ value & Marginal & Very long & $p$ value \\
\hline $0-8-\mathrm{km}$ shear $\left(\mathrm{m} \mathrm{s}^{-1}\right)$ & 28.33 & 33.23 & 0.0093 & 28.61 & 33.42 & 0.0204 & 26.79 & 29.69 & 0.2240 \\
\hline $0-3-\mathrm{km}$ shear $\left(\mathrm{m} \mathrm{s}^{-1}\right)$ & 15.32 & 15.97 & 0.5761 & 16.38 & 20.21 & 0.0126 & 15.91 & 18.66 & 0.1111 \\
\hline Effective shear $\left(\mathrm{m} \mathrm{s}^{-1}\right)$ & 22.96 & 25.29 & 0.2240 & 23.60 & 29.08 & 0.0040 & 22.81 & 27.02 & 0.1031 \\
\hline $0-3-\mathrm{km} \mathrm{SRH}\left(\mathrm{m}^{2} \mathrm{~s}^{-2}\right)$ & 182.18 & 169.05 & 0.9642 & 246.89 & 355.69 & 0.0295 & 278.57 & 351.90 & 0.0884 \\
\hline
\end{tabular}

Maddox 1976; Brooks et al. 1994; Rasmussen and Blanchard 1998; Rasmussen 2003; Parker 2014; Wade et al. 2018; Coffer and Parker 2019; Coniglio and Parker 2020). In the present dataset, the evolution in some of these parameters also appears to be relevant, as described below.

Tornado reports were observed throughout the lifetimes of the supercells in this dataset, and were associated with a wide range of buoyancy values, though the magnitude of CAPE tended to decrease for tornadoes observed near the end of a life cycle (Fig. 14a), consistent with previously identified diurnal trends (cf. Figs. 3-5). Changes in CAPE leading up to tornado reports predominately fell within the $\pm 50 \%$ range, with data most tightly clustered near zero percent changes (Fig. 14b). Thus, there are fairly equivalent numbers of tornadoes produced in environments where increases or decreases of CAPE are occurring, respectively, prior to tornadogenesis, suggesting that shifts in buoyancy are less of a contributing factor. For convective inhibition, no substantial trends in values occur for tornadoes early versus later in a supercell's lifetime (Fig. 14c). Changes in MLCIN leading up to tornado production were largely centered around zero, though a slight upward trend (i.e., increases in inhibition) over time is evident where the data is most strongly clustered (Fig. 14d). Tornado production as a function of MLLCL heights had little variation over time except for the few cases occurring near the very end of a storm's lifetime (Fig. 14e), with fairly small changes (most falling within $\pm 20 \%$ ) leading up to the tornado report, but a slight bias toward small decreases in MLCL height (Fig. 14f). Overall, thermodynamic changes in the environment do not appear to substantially influence tornado production in this dataset.

In contrast, low-level shear and SRH parameters exhibited more apparent patterns in values at the time of and leading up to tornado reports. For example, there is the expected diurnal trend of larger values of $0-1-\mathrm{km}$ shear, SRH, and effective SRH over time, likely related to the development of the nocturnal low-level jet (Figs. 15a,c,e). When examining changes in low-level shear and SRH leading up to tornado production
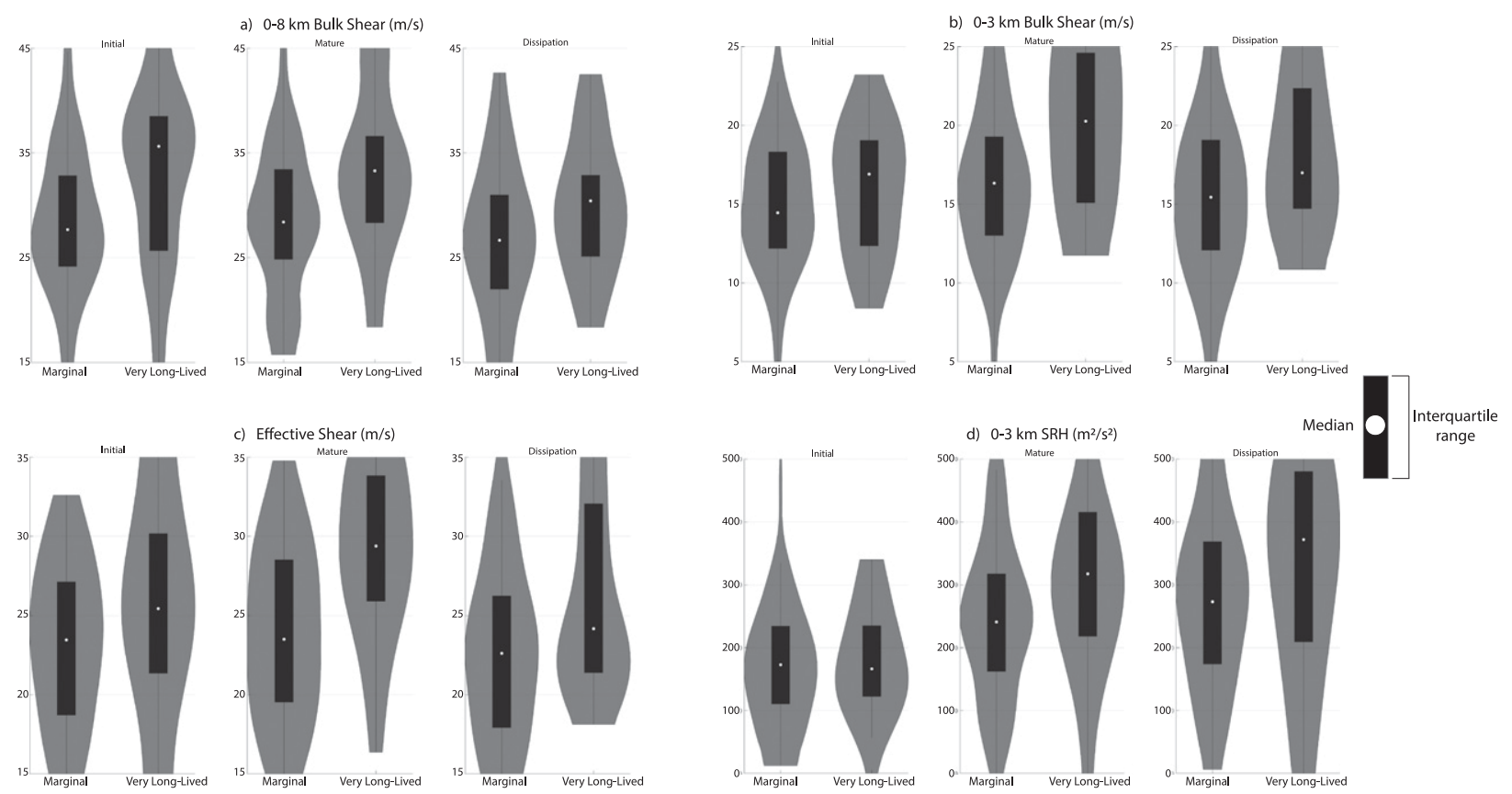

FIG. 12. Comparisons of select maturity-relative bulk wind shear and SRH parameters for marginally long-lived ( $\geq 4 \mathrm{~h}$ but $<5 \mathrm{~h}$ ) vs very long-lived $(\geq 7 \mathrm{~h})$ supercell environments. 


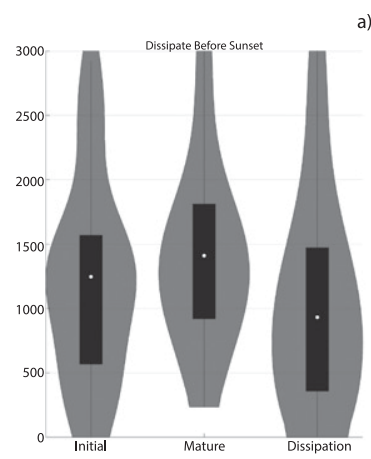

a) MLCAPE $(J / \mathrm{kg})$

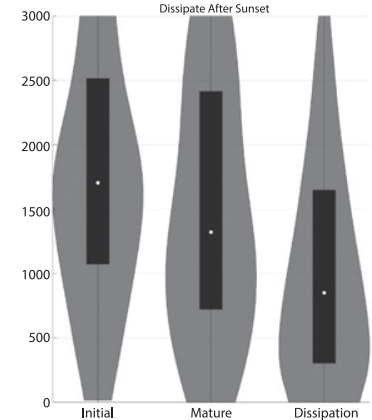

c) 0-1 km Bulk Shear $(\mathrm{m} / \mathrm{s})$
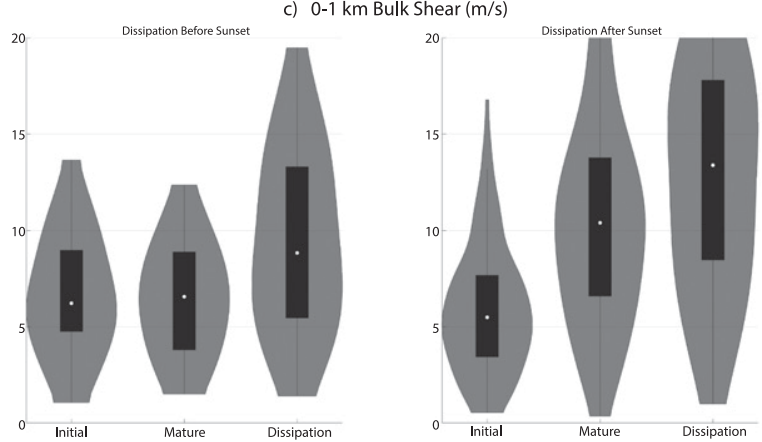

e) Effective Shear $(\mathrm{m} / \mathrm{s})$
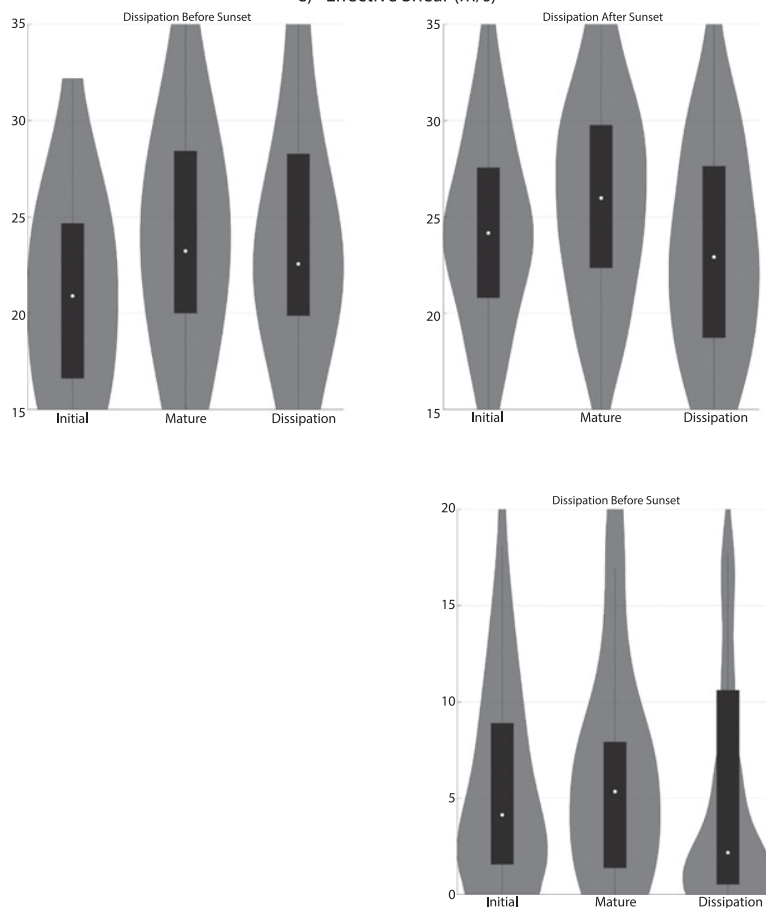

b) $\operatorname{MLCIN}(\mathrm{J} / \mathrm{kg})$
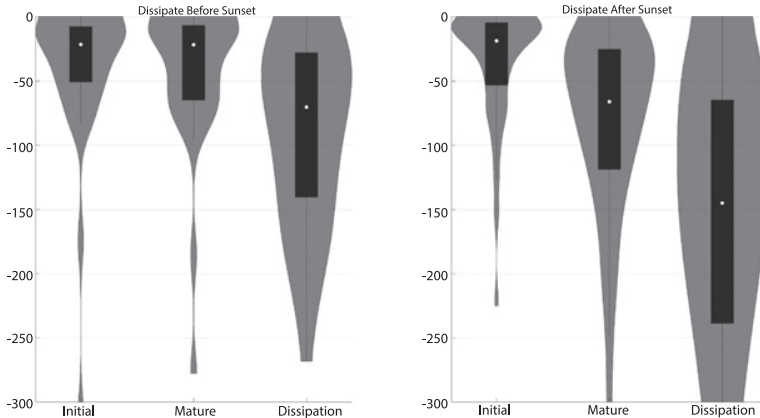

d) $0-8 \mathrm{~km}$ Bulk Shear $(\mathrm{m} / \mathrm{s})$
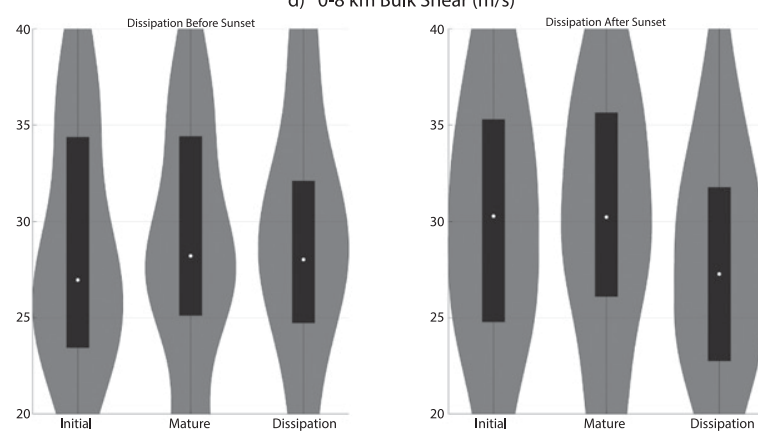

f) Effective $S R H\left(m^{2} / s^{2}\right)$
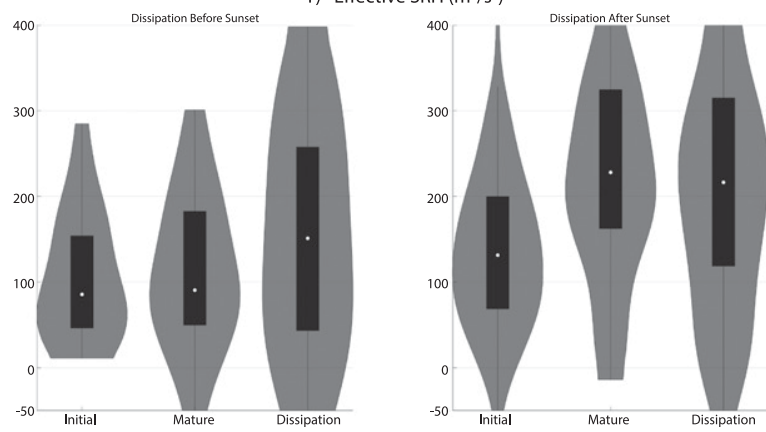

g) $\mathrm{SCP}$

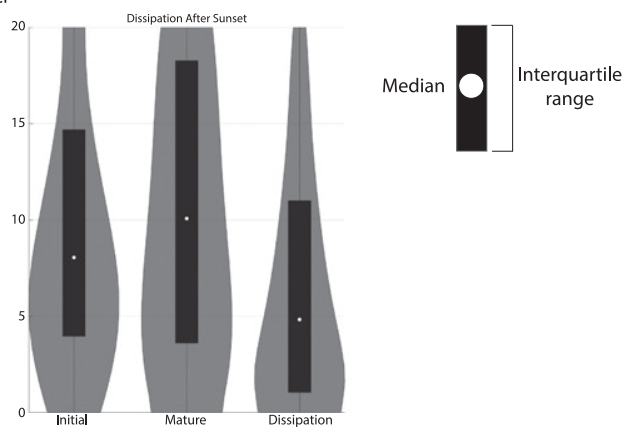

FIG. 13. Comparisons of evolution of select maturity-relative parameters for supercells that dissipated before vs after sunset (as labeled).

(Figs. 15b,d,f), there are two features to note. First, the sign of these changes is clustered around positive values (i.e., increases in shear and SRH), usually on the order of $25 \%-50 \%$. Second, the increases are most strongly clustered in the latter half of a supercell's lifetime, in line with when the diurnal trend and likely contribution of the nocturnal low-level jet is most apparent. These results are overall consistent with prior modeling work demonstrating that such increases in low-level shear 

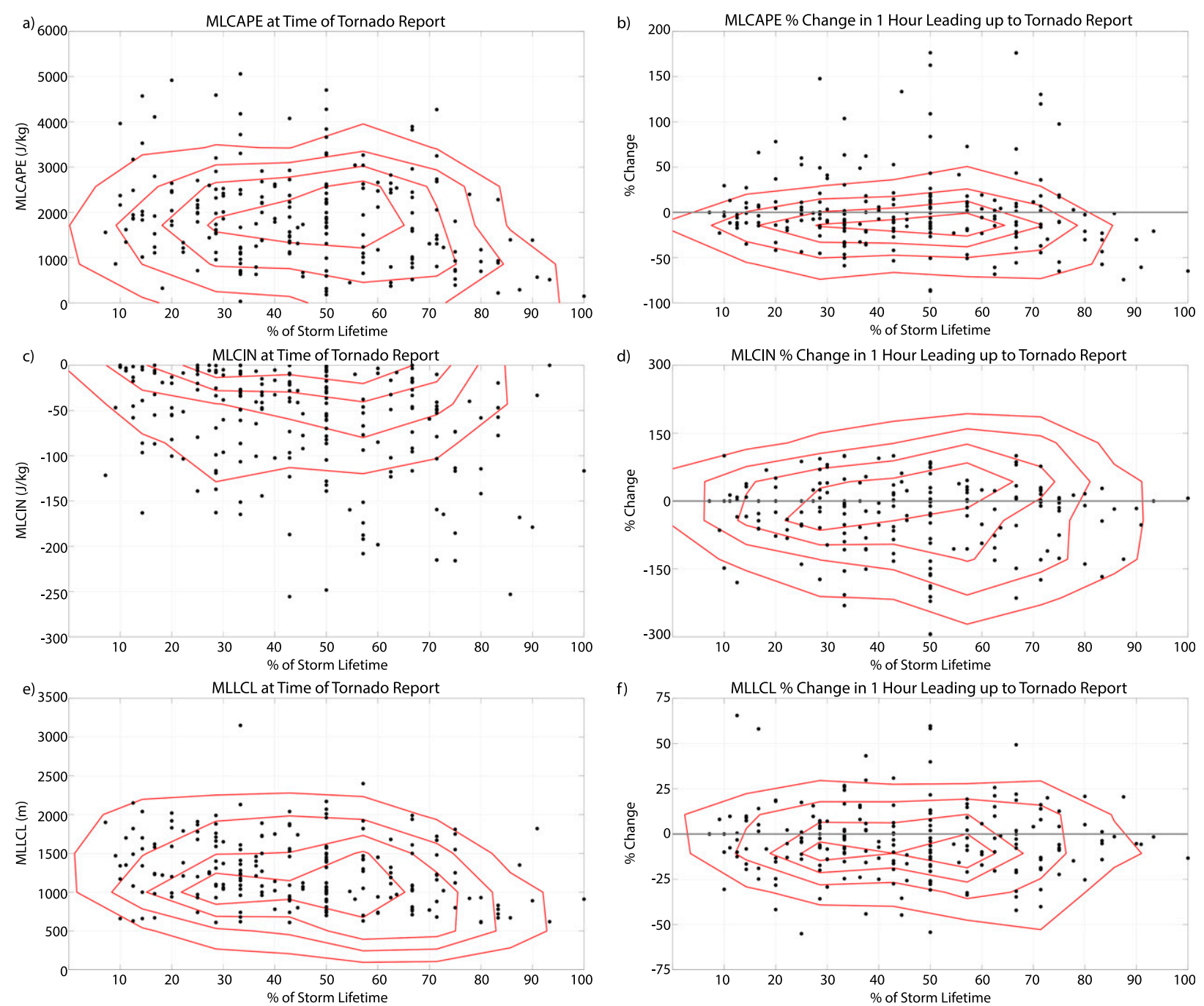

FIG. 14. Scatterplots of the timing of tornado reports (expressed as the percent of the storm lifetime passed at the time of the report) vs select near-inflow environment parameter values, including MLCAPE, MLCIN, and MLLCL. The contours represent the Gaussian kernel density estimates identifying where $25 \%$ (inner contour), $50 \%, 75 \%$, and $90 \%$ (outer contour) of the data are located. (a),(c),(e) Parameter values and density estimates at the time of the report, and (b),(d),(f) the percent change in a given parameter (relative to its starting value) in the $1 \mathrm{~h}$ leading up to the time of a tornado report (i.e., a positive percent change correlates to an increase in a given parameter in the hour leading up to the report).

promote enhanced dynamic accelerations and thus enhanced potential for tornado production (Coffer and Parker 2015).

The relationship between near-inflow environment characteristics and hail is largely poorly understood, with few good environmental predictors (Kumjian and Lombardo 2020). In the present dataset, thermodynamic parameters appear to be weakly correlated with hail reports, with a wide range of values associated with reports in combination with some apparent diurnal dependence; lower buoyancy combined with stronger inhibition and higher MLLFC heights tended to be present for reports occurring toward the end of a supercell's lifetime (Figs. 16a,c,e). Changes in these parameters in the hour leading up to hail production varied. MLCAPE and MLCIN changes mostly clustered near zero, with a slight bias toward small decreases in buoyancy (Figs. 16b,d). In contrast, changes in
LFC height, while also clustered near zero, largely exhibited small increases in altitude leading up to hail production (Fig. 16f).

Various bulk shear and storm-relative helicity parameters exhibited a wide range of values associated with hail reports, with generally little difference in values for reports occurring earlier versus later in a supercell's lifetime; 0-1-km bulk shear is the exception, with a slight upward trend in the latter half of a storm's lifetime, likely due to the development of the nocturnal low-level jet (Figs. 17a,c,e,g,i). The changes in bulk shear and SRH leading up to hail production are largely dependent on the vertical depth of interest. For example, hail reports are most strongly clustered around increases in $0-1-\mathrm{km}$ shear and SRH (Figs. 17b,h). This suggest that increases in 0-1-km shear and helicity may be relevant for hail production, as these 
a)
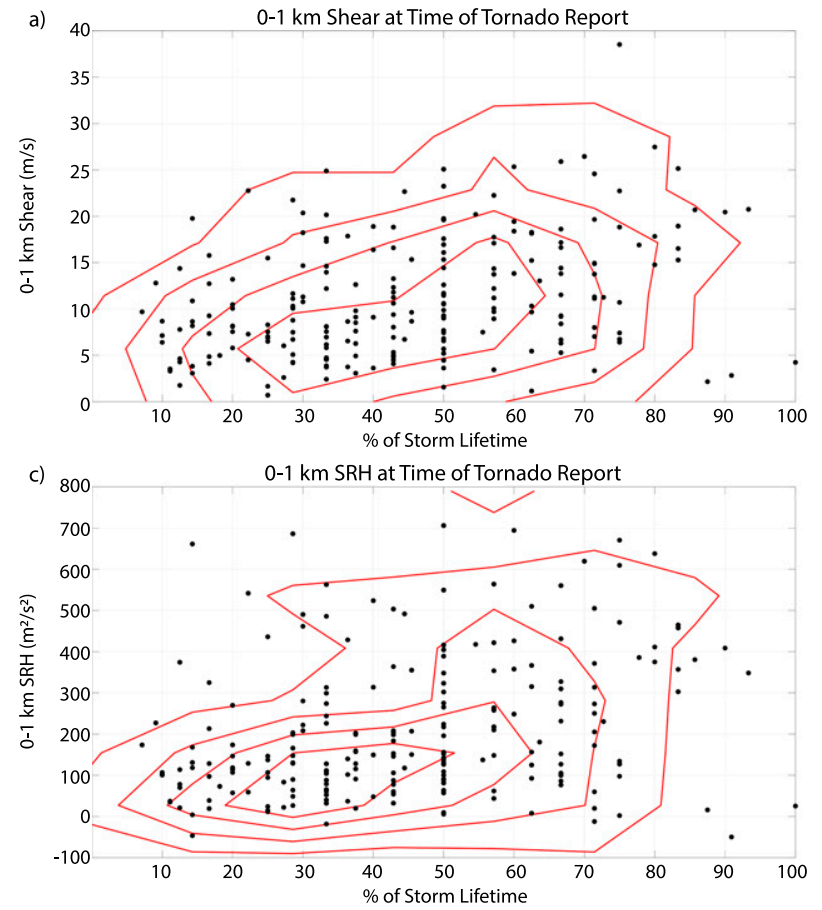

e) $900 \quad$ Effective SRH at Time of Tornado Report

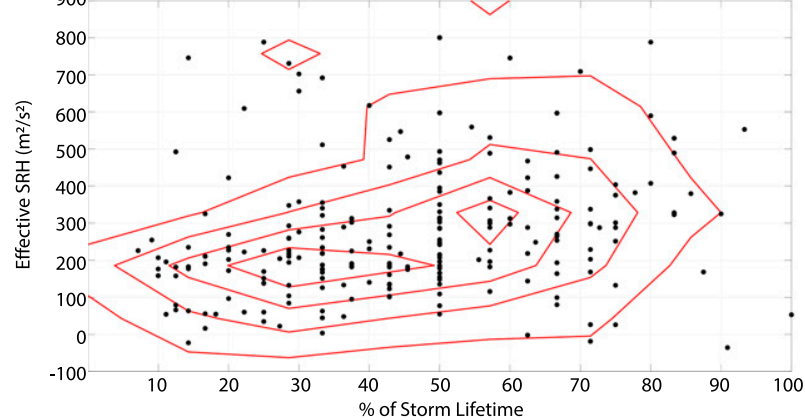

b) $300 \quad 0-1 \mathrm{~km}$ Shear $\%$ Change in 1 Hour Leading up to Tornado Report

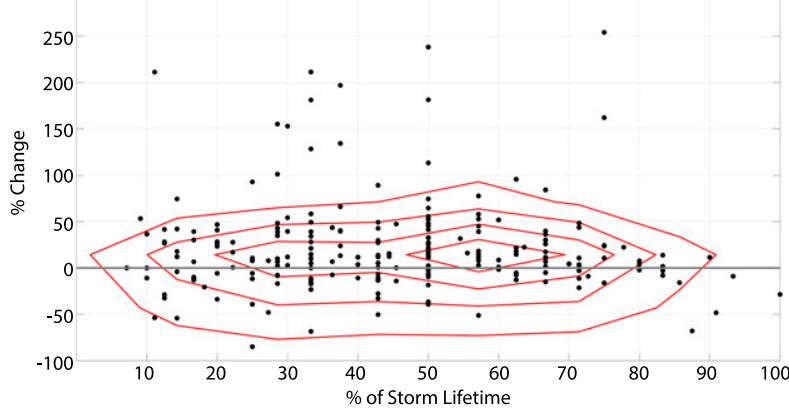

d)

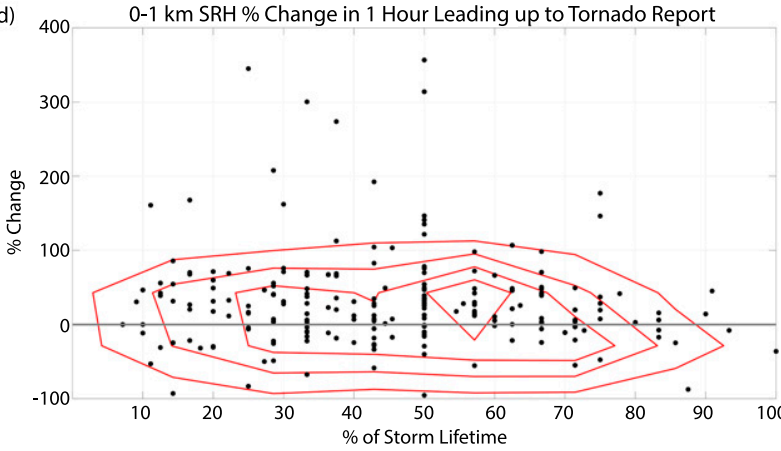

f) 200 Effective SRH \% Change in 1 Hour Leading up to Tornado Report

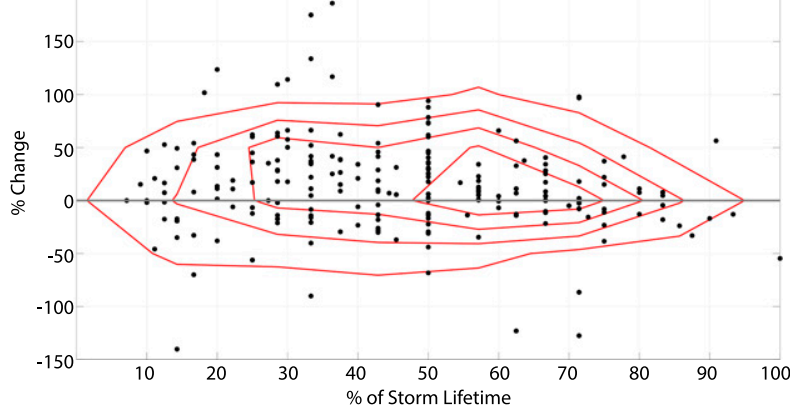

FIG. 15. As in Fig. 14, but for 0-1-km shear, 0-1-km SRH, and effective SRH.

changes could support the development of wider updrafts (e.g., Kumjian and Lombardo 2020). However, over deeper layers within $0-3$ or $0-6 \mathrm{~km}$, changes in shear and helicity tend to be evenly balanced between increases and decreases, or trend toward favoring small decreases leading up to hail reports (Figs. 17d,f,j).

Severe wind reports occurring in supercells are usually associated with the rear flank downdraft or occlusion downdraft, which are largely driven by dynamic pressure gradients (Markowski 2002). Other considerations, such as morphological changes (i.e., evolution into a high precipitation supercell or upscale growth into a cluster), can also contribute to the generation of severe wind gusts (e.g., Moller et al. 1994), but are not directly explored here. While there has been less investigation into the relationship between environment characteristics and severe wind production in supercells, other studies have demonstrated at least a few that are useful for wind reports generated by any type of convection, including CAPE, CIN, DCAPE, and
LCL heights (e.g., Gilmore and Wicker 1998; Kuchera and Parker 2006; Naylor et al. 2012; Taszarek et al. 2020). In the present dataset, severe wind reports occurring near the middle of a supercell's lifetime tend to be associated with the largest values of MLCAPE (Fig. 18a), though the majority of reports tend to have an even distribution of increases and decreases in buoyancy (Fig. 18b). There is also an apparent trend in MLCIN values, likely diurnal in nature, whereby wind reports occurring later are associated with larger inhibition as the low-level environment cools (Fig. 18c); there is also a slight trend toward increases in inhibition for wind reports occurring on the latter half of the supercell's lifetime (Fig. 18d). It is speculated that severe wind gusts follow from weakened support for a buoyant updraft, either via decreases in buoyancy or increases in inhibition, though this would need to be explored through additional analysis or idealized experiments. Last, severe wind gust production does not appear to be strongly a function of evaporative processes occurring as parcels descend below the cloud base. As 

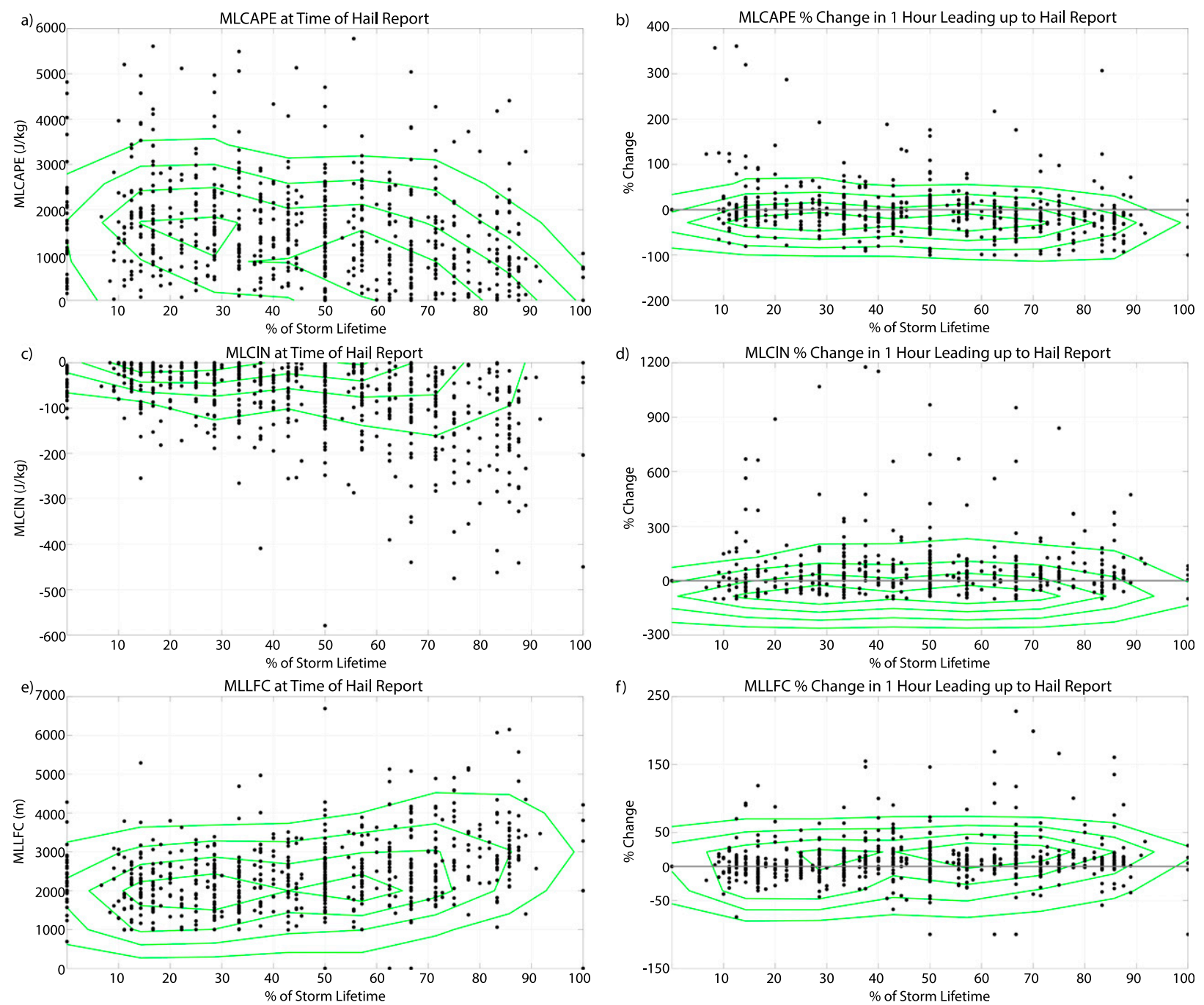

FIG. 16. As in Fig. 14, but for select thermodynamic parameters relevant to hail production.

evident in Figs. 18e-h, a variety of MLLCL and DCAPE values are associated with severe winds; notably, the relative changes in these parameters are fairly equally distributed between small increases and decreases prior to the generation of the severe wind gust.

\section{Summary and future work}

This study builds upon a growing body of work demonstrating and quantifying the effects of environmental variability on the evolution and behavior of severe convection. Focusing on these effects in supercell thunderstorms, a total of 147 long-lived supercells (with mesocyclones present for at least $4 \mathrm{~h}$ ) were identified and tracked throughout their lifetimes, providing an updated, storm-following perspective to the original Bunkers et al. (2006a,b) studies. The Lagrangian framework produced a series of near-storm RUC/RAP model proximity soundings that were generated at each hour and then used to calculate a variety of common forecasting parameters.
Severe weather reports associated with each supercell were also gathered, with representative environments assigned to each report.

The climatological characteristics of the long-lived supercells in this dataset were largely consistent with prior findings in Bunkers et al. (2006a), with both monthly and diurnal occurrence strongly correlated with seasonal and daytime heating (Fig. 2). The diurnal cycle is particularly evident in the timing of supercell initiation and dissipation (Fig. 3), severe weather production (Fig. 4), and in a number of thermodynamic and kinematic parameters (Figs. 5-7). The dominance of this signal further extends to the evolution in distributions of sounding parameters binned based on storm maturity (Figs. 9-10). Overall, the near-storm environment notably shifts throughout the lifetime of supercells, marked by decreases in CAPE, increases in CIN, and increases in low-level shear and SRH; these trends are likely connected to supercells frequently occurring near the nocturnal transition with attendant cooling and the development of the low-level jet. Notably, 

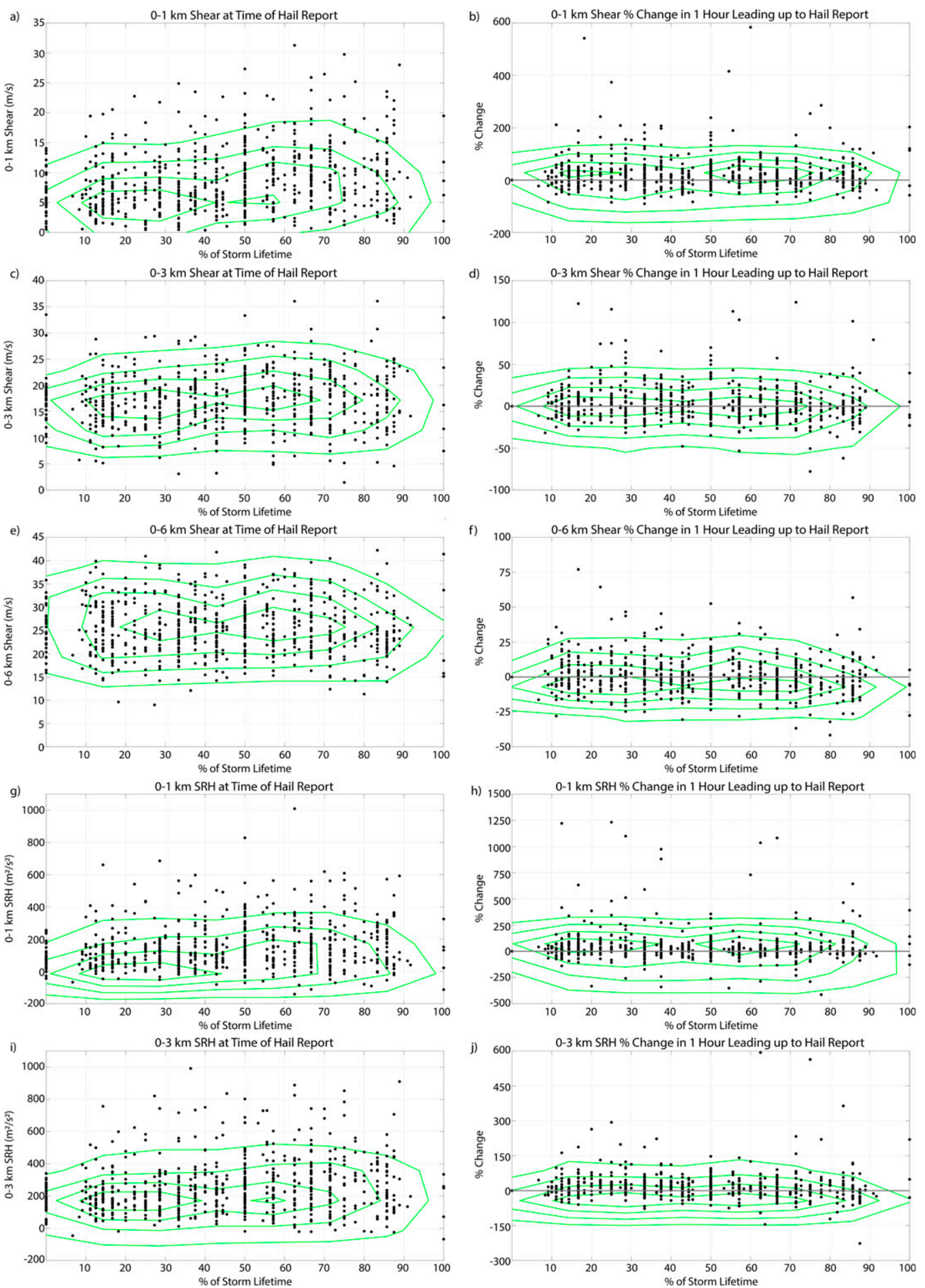

FIG. 17. As in Fig. 14, but for select bulk wind shear and SRH parameters relevant to hail production.

the dissipation stage is marked by a decrease in SCP, largely driven by reductions in buoyancy and increases in inhibition. Small decreases in deep-layer and effective shear were also present, but their physical significance is likely minimal.
The influence of environmental variability was further explored through examination of two subsets of cases. The first compared the environments of marginally long-lived (mesocyclones persisting for less than $5 \mathrm{~h}$ ) versus very long-lived 

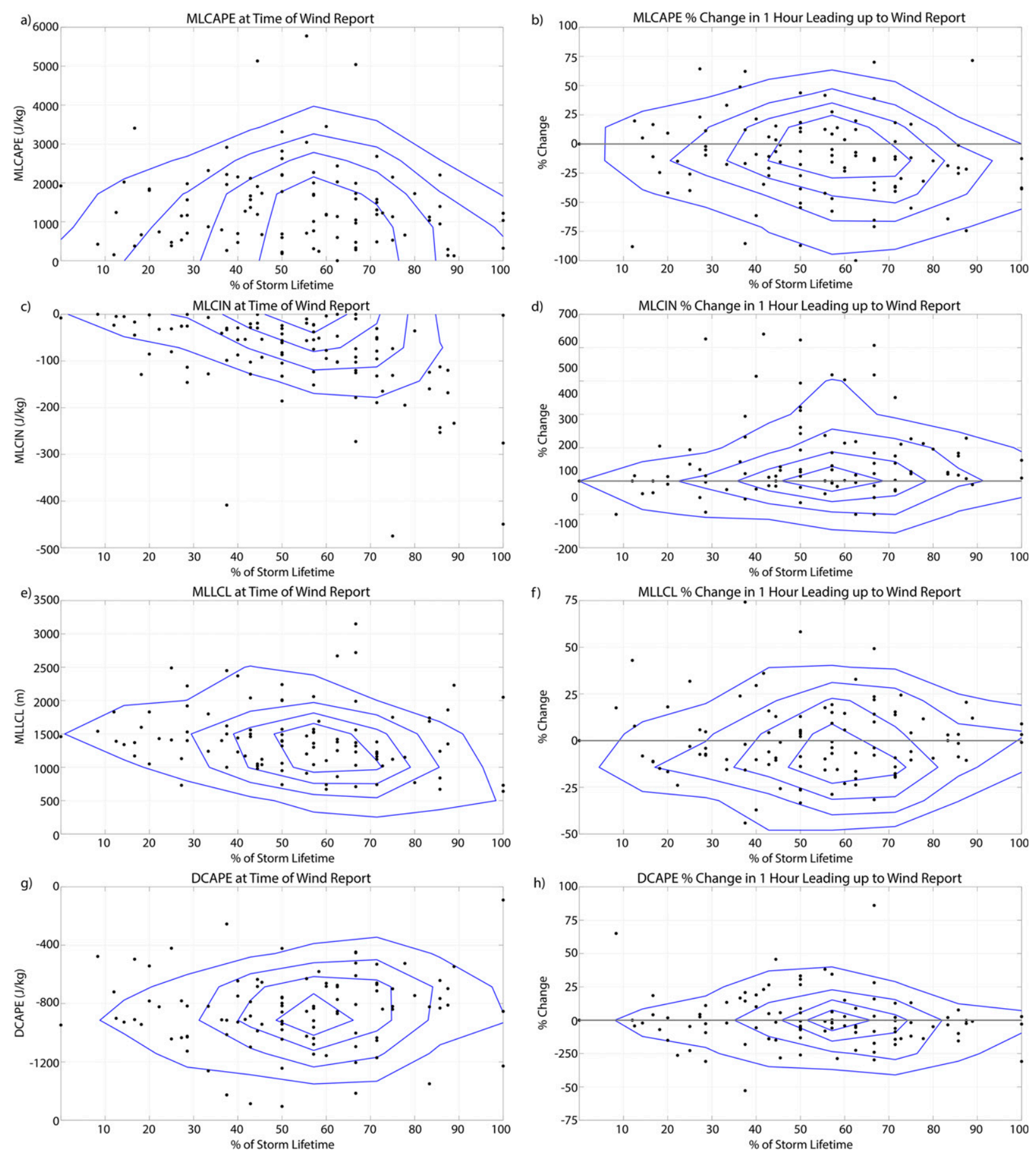

FIG. 18. As in Fig. 14, but for select parameters relevant to severe wind gust production.

supercells (mesocyclones persisting for at least $7 \mathrm{~h}$ ). Notably, the initial and mature environments of the very long-lived supercells contained significantly larger deep-layer shear, suggesting stronger dynamical support for supercell maintenance (e.g., as in Bunkers et al. 2006b). However, environmental changes also appeared to be important in distinguishing the groups. Though trends were largely similar, there were greater increases between the initial and mature environments for very long-lived supercells in key parameters such as low-level shear and SRH (Table 2; Fig. 12) that could contribute to sustained updraft rotation and storm maintenance. The second subset comparison focused on supercells that dissipated before versus after sunset to identify any nondiurnal effects, namely, whether the evolution in the near-storm environment differed. Though 
the magnitude of thermodynamic and kinematic parameters varied among the groupings due to their existence at varying times of day, overall environmental trends from initial to dissipation stages were similar (Fig. 13).

Variations in the near-storm environment were also found to be connected to severe weather production. For example, tornado reports tended to be preceded by increases in lowlevel shear and SRH (Fig. 15), suggesting that such changes are important for enhancing low-level updraft strength and rotation to support tornadogenesis, consistent with prior modeling work by Coffer and Parker (2015). Increases in these parameters were also correlated with hail production (Fig. 17), in line with other studies demonstrating that changes in the wind profile influence residence times and impact hail growth (Kumjian and Lombardo 2020). Severe wind gusts were more commonly reported following increases in MLCIN, particularly for those occurring later in a storm's lifetime (Fig. 18d).

This work represents an initial examination seeking to better understand the interplay between storms and their response to environmental variability, with many avenues left to explore. For example, a series of idealized modeling simulations would be useful to identify thresholds of changes in thermodynamic and kinematic quantities that lead to differences in supercell longevity, severe weather production, or even supercell morphology (e.g., Warren et al. 2017; Gropp and Davenport 2018; Sirico 2020). These tests could also examine how the governing processes within supercells are affected. As demonstrated by Davenport et al. (2019), incorporating changes in the background environment via base-state substitution would be a fruitful approach for ensuring such simulations are more realistic in nature.

Acknowledgments. Matt Gropp assisted with visualizing supercell storm tracks shown in Fig. 1. Thorough and constructive reviews from Bryan Smith, two anonymous reviewers, and the editor significantly improved this manuscript. Discussions with colleagues and graduate students at UNC Charlotte are also gratefully acknowledged.

Data availability statement. All radar and model data used for this study are openly available from the National Centers for Environmental information via the Archive Information Request System at https://www.ncei.noaa.gov/has/HAS.DsSelect. The Storm Data reports are publicly available at https:// www.spc.noaa.gov/wcm/\#data. The list of supercell cases and the model near-inflow latitude/longitude coordinates used can be accessed at this repository: https://doi.org/ 10.6084/m9.figshare.14230433.v1.

\section{REFERENCES}

Allen, J., M. Kumjian, C. Nixon, R. Jewell, B. Smith, and R. Thompson, 2020: Forecast parameters for U.S. hail occurrence and size. 30th Conf. on Weather Analysis and Forecasting, Boston, MA, Amer. Meteor. Soc., 1A.4, https://ams.confex.com/ams/ 2020Annual/meetingapp.cgi/Paper/368490.

Anderson-Frey, A., Y. Richardson, A. Dean, R. Thompson, and B. Smith, 2016: Investigation of near-storm environments for tornado events and warnings. Wea. Forecasting, 31, 1771-1790, https://doi.org/10.1175/WAF-D-16-0046.1.
Andra, D., 1997: The origin and evolution of the WSR-88D mesocyclone recognition nomogram. Preprints, 28th Conf. on Radar Meteorology, Austin, TX, Amer. Meteor. Soc., 364-365. Benjamin, S., T. Smirnova, J. Brown, G. Grell, and R. Bleck, 2004: Mesoscale weather prediction with the RUC hybrid isentropic-terrain-following coordinate model. Mon. Wea. Rev., 132, 473-494, https://doi.org/10.1175/1520-0493(2004) 132<0473:MWPWTR>2.0.CO;2.

—, and Coauthors, 2016: A North American hourly assimilation and model forecast cycle: The Rapid Refresh. Mon. Wea. Rev., 144, 1669-1694, https://doi.org/10.1175/MWR-D-15-0242.1.

Bosart, L., A. Seimon, K. LaPenta, and M. Dickinson, 2006: Supercell tornadogenesis over complex terrain: The Great Barrington, Massachusetts, tornado on 29 May 1995. Wea. Forecasting, 21, 897-922, https://doi.org/10.1175/WAF957.1.

Boustead, J., B. Mayes, W. Gagan, J. Leighton, G. Phillips, and P. Schumacher, 2013: Discriminating environmental conditions for significant warm sector and boundary tornadoes in parts of the Great Plains. Wea. Forecasting, 28, 1498-1523, https://doi.org/10.1175/WAF-D-12-00102.1.

Brooks, H., and C. Doswell, 2001: Some aspects of the international climatology of tornadoes by damage classification. Atmos. Res., 56, 191-201, https://doi.org/10.1016/S0169-8095(00)00098-3.

_ C. A. Doswell III, and J. Cooper, 1994: On the environments of tornadic and nontornadic mesocyclones. Wea. Forecasting, 9, 606-618, https://doi.org/10.1175/1520-0434(1994)009<0606: OTEOTA $>2.0 . \mathrm{CO} ; 2$.

— C. Doswell III, M. Carr, and J. Rutherford, 1996: Preliminary analysis of VORTEX-95 soundings. Preprints, 19th Conf. on Severe Local Storms, San Francisco, CA, Amer. Meteor. Soc., 133-136.

_- C. Doswell, and M. Kay, 2003: Climatological estimates of local daily tornado probability for the United States. Wea. Forecasting, 18, 626-640, https://doi.org/10.1175/ 1520-0434(2003)018<0626:CEOLDT > 2.0.CO;2.

Bunkers, M., B. Klimowski, J. Zeitler, R. Thompson, and M. Weisman, 2000: Predicting supercell motion using a new hodograph technique. Wea. Forecasting, 15, 61-79, https://doi.org/10.1175/15200434(2000)015<0061:PSMUAN >2.0.CO;2.

— - M. Hjelmfelt, and P. Smith, 2006a: An observational examination of long-lived supercells. Part I: Characteristics, evolution, and demise. Wea. Forecasting, 21, 673-688, https:// doi.org/10.1175/WAF949.1.

- J. Johnson, L. Czepyha, J. Grzywacz, B. Klimowski, and M. Hjelmfelt, 2006b: An observational examination of longlived supercells. Part II: Environmental conditions and forecasting. Wea. Forecasting, 21, 689-714, https://doi.org/10.1175/ WAF952.1.

Burgess, D., and L. Lemon, 1991: Characteristics of mesocyclones detected during a NEXRAD test. Preprints, 25th Int. Conf. on Radar Meteorology, Paris, France, Amer. Meteor. Soc., 39-42.

Changnon, S., and J. Burroughs, 2003: The tristate hailstorm: The most costly on record. Mon. Wea. Rev., 131, 1734-1739, https:// doi.org/10.1175//2549.1.

Coffer, B., and M. Parker, 2015: Impacts of increasing low-level shear on supercells during the early evening transition. Mon. Wea. Rev., 143, 1945-1969, https://doi.org/10.1175/MWR-D14-00328.1.

— and - 2019: Using near-ground storm relative helicity in supercell forecasting. Wea. Forecasting, 34, 1417-1435, https:// doi.org/10.1175/WAF-D-19-0115.1.

— of tornadic and nontornadic environments in the United 
States and Europe from ERA5 reanalyses. Wea. Forecasting, 35, 2621-2638, https://doi.org/10.1175/WAF-D-20-0153.1.

Cohen, A., M. Coniglio, S. Cavallo, and H. Brooks, 2015: A review of planetary boundary layer parameterization schemes and their sensitivity in simulating southeastern U.S. cold season severe weather environments. Wea. Forecasting, 30, 591-612, https://doi.org/10.1175/WAF-D-14-00105.1.

Coniglio, M., 2012: Verification of RUC 0-1-h forecasts and SPC mesoscale analyses using VORTEX2 environments. Wea. Forecasting, 27, 667-683, https://doi.org/10.1175/WAF-D-1100096.1.

— ronments from three decades of targeted radiosonde observations. Mon. Wea. Rev., 148, 4893-4915, https://doi.org/ 10.1175/MWR-D-20-0105.1.

Davenport, C., and M. Parker, 2015a: Impact of environmental heterogeneity on the dynamics of a dissipating supercell thunderstorm. Mon. Wea. Rev., 143, 4244-4277, https://doi.org/ 10.1175/MWR-D-15-0072.1.

— , and — 2015b: Observations of the 9 June 2009 dissipating supercell from VORTEX2. Wea. Forecasting, 30, 368-388, https://doi.org/10.1175/WAF-D-14-00087.1.

_ C. Ziegler, and M. Biggerstaff, 2019: Creating a more realistic idealized supercell thunderstorm evolution via incorporation of base-state environmental variability. Mon. Wea. Rev., 147, 4177-4198, https://doi.org/10.1175/MWR-D-18-0447.1.

Doswell, C., and D. Burgess, 1988: On some issues of United States tornado climatology. Mon. Wea. Rev., 116, 495-501, https:// doi.org/10.1175/1520-0493(1988)116<0495:OSIOUS >2.0.CO;2.

_ temperature correction on CAPE calculations. Wea. Forecasting, 9, 625-629, https://doi.org/10.1175/1520-0434(1994)009<0625: TEONTV $>2.0 . \mathrm{CO} ; 2$.

Edwards, R., J. Allen, and G. Carbin, 2018: Reliability and climatological impacts of convective wind estimations. J. Appl. Meteor. Climatol., 57, 1825-1845, https://doi.org/ 10.1175/JAMC-D-17-0306.1.

Frame, J., and P. Markowski, 2013: Dynamical influences of anvil shading on simulated supercell thunderstorms. Mon. Wea. Rev., 141, 2802-2820, https://doi.org/10.1175/MWR-D-12-00146.1.

Gagne, D., A. McGovern, J. Basara, and R. Brown, 2012: Tornadic supercell environments analyzed using surface and reanalysis data: A spatiotemporal relational data-mining approach. J. Appl. Meteor. Climatol., 51, 2203-2217, https://doi.org/ 10.1175/JAMC-D-11-060.1.

Gilmore, M., and L. Wicker, 1998: The influence of midtropospheric dryness on supercell morphology and evolution. Mon. Wea. Rev., 126, 943-958, https://doi.org/10.1175/15200493(1998)126<0943:TIOMDO>2.0.CO;2.

Glass, F., and M. Britt, 2002: The historic Missouri-Illinois high precipitation supercell of 10 April 2001. Preprints, 21st Conf. on Severe Local Storms, San Antonio, TX, Amer. Meteor. Soc., P3.3, https://ams.confex.com/ams/SLS_WAF_ NWP/techprogram/paper_47280.htm.

Gropp, M., and C. Davenport, 2018: The impact of the nocturnal transition on the lifetime and evolution of supercell thunderstorms in the Great Plains. Wea. Forecasting, 33, 1045-1061, https://doi.org/10.1175/WAF-D-17-0150.1.

Hales, J., 1988: Improving the watch/warning program through use of significant even data. Preprints, 15th Conf. on Severe Local Storms, Baltimore, MD, Amer. Meteor. Soc., 165-168.

Hartigan, J., R. Warren, J. Soderholm, and H. Richter, 2021: Simulated changes in storm morphology associated with a sea-breeze air mass. Mon. Wea. Rev., 149, 333-351, https:// doi.org/10.1175/MWR-D-20-0069.1.

Hastings, R., and Y. Richardson, 2016: Long-term morphological changes in simulated supercells following mergers with nascent supercells in directionally varying shear. Mon. Wea. Rev., 144, 471-499, https://doi.org/10.1175/MWR-D15-0193.1.

Kerr, B., and G. Darkow, 1996: Storm-relative winds and helicity in the tornadic thunderstorm environment. Wea. Forecasting, 11, 489-505, https://doi.org/10.1175/15200434(1996)011<0489:SRWAHI >2.0.CO;2.

Kis, A., and J. Straka, 2010: Nocturnal tornado climatology. Wea. Forecasting, 25, 545-561, https://doi.org/10.1175/2009WAF2222294.1.

Klees, A., Y. Richardson, P. Markowski, C. Weiss, J. Wurman, and K. Kosiba, 2016: Comparison of the tornadic and nontornadic supercells intercepted by VORTEX2 on 10 June 2010. Mon. Wea. Rev., 144, 3201-3231, https://doi.org/10.1175/MWR-D15-0345.1.

Koch, S., R. Ware, H. Jiang, and Y. Xie, 2016: Rapid mesoscale environmental changes accompanying genesis of an unusual tornado. Wea. Forecasting, 31, 763-786, https://doi.org/10.1175/ WAF-D-15-0105.1.

Kuchera, E., and M. Parker, 2006: Severe convective wind environments. Wea. Forecasting, 21, 595-612, https://doi.org/10.1175/ WAF931.1.

Kumjian, M., and K. Lombardo, 2020: A hail growth trajectory model for exploring the environmental controls on hail size: Model physics and idealized tests. J. Atmos. Sci., 77, 27652791, https://doi.org/10.1175/JAS-D-20-0016.1.

Letkewicz, C., A. French, and M. Parker, 2013: Base-state substitution: An idealized modeling technique for approximating environmental variability. Mon. Wea. Rev., 141, 3062-3086, https://doi.org/10.1175/MWR-D-12-00200.1.

Li, F., D. Chavas, K. Reed, and D. T. Dawson II, 2020: Climatology of severe local storm environments and synoptic-scale features over North America in ERA5 reanalysis and CAM6 simulation. J. Climate, 33, 8339-8365, https://doi.org/10.1175/JCLID-19-0986.1.

Maddox, R., 1976: An evaluation of tornado proximity wind and stability data. Mon. Wea. Rev., 104, 133-142, https://doi.org/ 10.1175/1520-0493(1976)104<0133:AEOTPW >2.0.CO;2.

_ 1993: Diurnal low-level wind oscillation and storm-relative helicity. The Tornado: Its Structure, Dynamics, Prediction, and Hazards, Geophys. Monogr., Vol. 79, Amer. Geophys. Union, 591-598.

- L. Hoxit, and C. Chappell, 1980: A study of tornadic thunderstorm interactions with thermal boundaries. Mon. Wea. Rev., 108, 322-336, https://doi.org/10.1175/1520-0493(1980) $108<0322$ :ASOTTI $>2.0$. CO;2.

Magee, K., and C. Davenport, 2020: An observational analysis quantifying the distance of supercell-boundary interactions in the Great Plains. J. Oper. Meteor., 8, 15-38, https://doi.org/ 10.15191/nwajom.2020.0802.

Markowski, P., 2002: Hook echoes and rear-flank downdrafts: A review. Mon. Wea. Rev., 130, 852-876, https://doi.org/10.1175/ 1520-0493(2002)130<0852:HEARFD>2.0.CO;2.

- , and Y. Richardson, 2007: Observations of vertical wind shear heterogeneity in convective boundary layers. Mon. Wea. Rev., 135, 843-861, https://doi.org/10.1175/MWR3334.1.

_, E. Rasmussen, and J. Straka, 1998a: The occurrence of tornadoes in supercells interacting with boundaries during VORTEX-95. Wea. Forecasting, 13, 852-859, https://doi.org/10.1175/ 1520-0434(1998)013<0852:TOOTIS >2.0.CO;2. 
_ J. Straka, E. Rasmussen, and D. Blanchard, 1998b: Variability of storm-relative helicity during VORTEX. Mon. Wea. Rev., 126, 2959-2971, https://doi.org/10.1175/1520-0493(1998) $126<2959$ :VOSRHD $>2.0 . \mathrm{CO} ; 2$.

Mead, C., and R. Thompson, 2011: Environmental characteristics associated with nocturnal significant-tornado events in the central and southern Great Plains. Electron. J. Severe Storms Meteor., 6 (6), https://www.spc.noaa.gov/publications/mead/ noctors.pdf.

Moller, A., C. Doswell III, M. Foster, and G. Woodall, 1994: The operational recognition of supercell thunderstorm environments and storm structures. Wea. Forecasting, 9, 327-347, https://doi.org/ 10.1175/1520-0434(1994)009<0327:TOROST >2.0.CO;2.

Naylor, J., M. Askelson, and M. Gilmore, 2012: Influence of lowlevel thermodynamic structure on the downdraft properties of simulated supercells. Mon. Wea. Rev., 140, 2575-2589, https:// doi.org/10.1175/MWR-D-11-00200.1.

Nowotarski, C., and P. Markowski, 2016: Modifications to the nearstorm environment induced by simulated supercell thunderstorms. Mon. Wea. Rev., 144, 273-293, https://doi.org/10.1175/ MWR-D-15-0247.1.

Parker, M., 2014: Composite VORTEX2 supercell environments from near-storm soundings. Mon. Wea. Rev., 142, 508-529, https://doi.org/10.1175/MWR-D-13-00167.1.

Potvin, C., K. Elmore, and S. Weiss, 2010: Assessing the impacts of proximity sounding criteria on the climatology of significant tornado events. Wea. Forecasting, 25, 921-930, https://doi.org/ 10.1175/2010WAF2222368.1.

Rasmussen, E., 2003: Refined supercell and tornado forecast parameters. Wea. Forecasting, 18, 530-535, https://doi.org/10.1175/ 1520-0434(2003)18<530:RSATFP $>2.0$.CO;2.

-_, and D. Blanchard, 1998: A baseline climatology of sounding-derived supercell and tornado forecast parameters. Wea. Forecasting, 13, 1148-1164, https://doi.org/ 10.1175/1520-0434(1998)013<1148:ABCOSD>2.0.CO;2.

_ S. Richardson, J. Straka, and P. M. D. Blanchard, 2000: The association of significant tornadoes with a baroclinic boundary on 2 June 1995. Mon. Wea. Rev., 128, 174-191, https://doi.org/ 10.1175/1520-0493(2000)128<0174:TAOSTW >2.0.CO;2.

Reames, L., 2017: Diurnal variations in severe weather forecast parameters of Rapid Update Cycle-2 tornado proximity environments. Wea. Forecasting, 32, 743-761, https://doi.org/ 10.1175/WAF-D-16-0029.1.

Richardson, Y., 2016: Examining storm evolution within idealized heterogeneous environments. 28th Conf. on Severe Local Storms, Portland, OR, Amer. Meteor. Soc., 6A.3, https:// ams.confex.com/ams/28SLS/webprogram/Paper301810.html.

, K. Droegemeier, and R. Davies-Jones, 2007: The influence of horizontal environmental variability on numerically simulated convective storms. Part I: Variations in vertical shear. Mon. Wea. Rev., 135, 3429-3455, https://doi.org/10.1175/MWR3463.1. Sirico, R., 2020: Investigation of the environmental influences related to the precipitation structure of supercell thunderstorms and their evolution. M.S. thesis, Dept. of Geography and Earth Sciences, University of North Carolina at Charlotte, $137 \mathrm{pp}$.

Smith, B., R. Thompson, J. Grams, C. Broyles, and H. Brooks, 2012: Convective modes for significant severe thunderstorms in the contiguous United States. Part I: Storm classification and climatology. Wea. Forecasting, 27, 1114-1135, https:// doi.org/10.1175/WAF-D-11-00115.1.

Taszarek, M., J. Allen, T. Púčik, K. Hoogewind, and H. Brooks, 2020: Severe convective storms across Europe and the United States. Part II: ERA5 environments associated with lightning, large hail, severe wind, and tornadoes. J. Climate, 33, $10263-$ 10 286, https://doi.org/10.1175/JCLI-D-20-0346.1.

Thompson, R., and R. Edwards, 2000: An overview of environmental conditions and forecast implications of the 3 May 1999 tornado outbreak. Wea. Forecasting, 15, 682-699, https:// doi.org/10.1175/1520-0434(2000)015<0682: AOOECA $>2.0 . \mathrm{CO} ; 2$.

, _ _ J. J. Hart, K. Elmore, and P. Markowski, 2003: Close proximity soundings within supercell environments obtained from the Rapid Update Cycle. Wea. Forecasting, 18, 1243-1261, https:// doi.org/10.1175/1520-0434(2003)018<1243:CPSWSE>2.0.CO;2.

—, C. Mead, and R. Edwards, 2007: Effective storm-relative helicity and bulk shear in supercell thunderstorm environments. Wea. Forecasting, 22, 102-115, https://doi.org/10.1175/ WAF969.1.

Wade, A., M. Coniglio, and C. Ziegler, 2018: Comparison of nearand far-field supercell inflow environments using radiosonde observations. Mon. Wea. Rev., 146, 2403-2415, https://doi.org/ 10.1175/MWR-D-17-0276.1.

Warren, R., H. Richter, H. Ramsay, S. Siems, and M. Manton, 2017: Impact of variations in upper-level shear on simulated supercells. Mon. Wea. Rev., 145, 2659-2681, https://doi.org/ 10.1175/MWR-D-16-0412.1.

Weiss, S., J. Hart, and P. Janish, 2002: An examination of severe thunderstorm wind report climatology: 1970-1999. Preprints, 21st Conf. on Severe Local Storms, San Antonio, TX, Amer. Meteor. Soc., 446-449.

Wilks, D., 2011: Statistical Methods in the Atmospheric Sciences. 3rd ed. International Geophysics Series, Vol. 100, Academic Press, 704 pp.

Ziegler, C., E. Mansell, J. Straka, D. MacGorman, and D. Burgess, 2010: The impact of spatial variations of lowlevel stability on the life cycle of a simulated supercell storm. Mon. Wea. Rev., 138, 1738-1766, https://doi.org/ 10.1175/2009MWR3010.1. 